\title{
PROJECTIVE SURFACE AUTOMORPHISMS OF POSITIVE TOPOLOGICAL ENTROPY FROM AN ARITHMETIC VIEWPOINT
}

\author{
SHU KAWAGUCHI
}

\begin{abstract}
Let $X$ be a smooth projective surface over a number field $K(\subset \mathbb{C})$, and $f$ : $X \rightarrow X$ an automorphism of positive topological entropy. In this paper, we show that there are only finitely many $f$-periodic curves on $X$. Then we define a height function $\widehat{h}_{D}$ corresponding to a certain nef and big $\mathbb{R}$-divisor $D$ on $X$ and transforming well relative to $f$, and deduce some arithmetic properties of $f$-periodic points and non $f$-periodic points.
\end{abstract}

\section{INTRODUCTION}

Let $X$ be a smooth projective surface over a number field $K \subset \mathbb{C}$, and $f: X \rightarrow X$ an automorphism.

For $x \in X(\bar{K})$, we denote by $O_{f}^{+}(x):=\left\{f^{n}(x) \mid n \geq 1\right\}$ the forward orbit of $x$ under $f$. We fix an ample line bundle $H$ on $X$ and take a height function $h_{H}: X(\bar{K}) \rightarrow \mathbb{R}$ corresponding to $H$. A point $x$ is said to be $f$-periodic if $f^{n}(x)=x$ for some positive integer $n$. Since $f$ is an automorphism, $x$ is $f$-periodic if and only if $O_{f}^{+}(x)$ is a finite set. If $O_{f}^{+}(x)$ is not a finite set, we estimate an arithmetic size of $O_{f}^{+}(x)$ by defining

$$
N_{f, x}^{+}(T):=\#\left\{y \in O_{f}^{+}(x) \mid h_{H}(y) \leq T\right\}
$$

for $T>0$.

We set $X_{\mathbb{C}}:=X \times_{\operatorname{Spec}(K)} \operatorname{Spec}(\mathbb{C})$, and write $f_{\mathbb{C}}: X_{\mathbb{C}} \rightarrow X_{\mathbb{C}}$ for the induced automorphism. Then $f_{\mathbb{C}}$ induces the linear transformation $f_{\mathbb{C}}^{*}$ on $H^{1,1}\left(X_{\mathbb{C}}, \mathbb{R}\right)$. Let $\lambda$ be the spectral radius of $f_{\mathbb{C}}^{*}$, i.e., the maximum of the absolute values of all the eigenvalues of $f_{\mathbb{C}}^{*}$. Note that $\lambda$ is equal to the first dynamical degree $\lambda_{1}\left(f_{\mathbb{C}}\right)$ of $f_{\mathbb{C}}$, defined by $\lambda_{1}\left(f_{\mathbb{C}}\right):=\lim _{n \rightarrow+\infty}\left\|f_{\mathbb{C}}^{n *}\right\|^{\frac{1}{n}}$ (see for example $[9, \S 1.4]$ and $[10])$.

In this paper, we prove the following assertion (a more precise form will be given below), in a sense generalizing [21].

Recall that a subset $S \subset X(\bar{K})$ is called a set of bounded height if there is a constant $c$ such that $h_{H}(s) \leq c$ for all $s \in S$.

Assertion A. Assume $\lambda>1$. Then

(1) there are a height function $\widehat{h}$ on $X(\bar{K})$ and a proper Zariski-closed subset $Z_{1}$ of $X$ such that $\widehat{h}$ transforms well relative to $f$ and such that $\widehat{h}$ satisfies the Northcott finiteness property on $\left(X \backslash Z_{1}\right)(\bar{K})$.

2000 Mathematics Subject Classification. 11G50, 14G05, 32H50.

Key words and phrases. height function, projective surface automorphism, rational point, periodic point, topological entropy. 
(2) There is a proper Zariski-closed subset $Z_{2}$ of $X$ such that

$$
\left\{x \in\left(X \backslash Z_{2}\right)(\bar{K}) \mid x \text { is } f \text {-periodic }\right\}
$$

is a set of bounded height.

(3) There is a proper Zariski-closed subset $Z_{3}$ of $X$ such that, if $x \in\left(X \backslash Z_{3}\right)(\bar{K})$ is a non $f$-periodic point, then

$$
\lim _{T \rightarrow+\infty} \frac{N_{f, x}^{+}(T)}{\log T}=\frac{1}{\log \lambda} .
$$

Before discussing Assertion A in more detail, we remark that (a similar) assertion is known to be true for some other $(X, f)$. We consider a modified assertion of Assertion A, in which $\lambda$ is replaced by the first dynamical degree $\lambda_{1}\left(f_{\mathbb{C}}\right)$, and $f$-periodicity by $f$-preperiodicity. (Here $x$ is said to be $f$-preperiodic if $O_{f}^{+}(x)$ is a finite set.)

The first case we remark is when $X$ is a smooth projective variety and $f: X \rightarrow X$ is a morphism such that $f^{*}(L) \sim d L$ for some ample divisor $L$ and $d>1$. This case is studied in Call-Silverman [6]. Here the modified assertion holds true with $Z_{1}=Z_{2}=Z_{3}=\emptyset$ by [6, Theorem 1.1 and Corollary 1.1]. We note that one can check $\lambda_{1}\left(f_{\mathbb{C}}\right)=d(>1)$ in this case, using $\lambda_{1}\left(f_{\mathbb{C}}\right)=\lim _{n \rightarrow+\infty}\left(\int_{X} f^{n *} \omega \wedge \omega^{\operatorname{dim} X_{\mathbb{C}}-1}\right)^{\frac{1}{n}}$ for any normalized Kähler form $\omega$ on $X_{\mathbb{C}}$ ([10, p. 314] and [9, Remark 1.17]). The second case is when $X$ is $\mathbb{P}^{2}$ and $f: \mathbb{P}^{2} \rightarrow \mathbb{P}^{2}$ is a birational extension of a polynomial automorphism of the affine plane $f_{0}: \mathbb{A}^{2} \rightarrow \mathbb{A}^{2}$. Then the modified assertion holds true with $Z_{1}=Z_{2}=Z_{3}=H_{\infty}$, where $H_{\infty}$ is the hyperplane at infinity, i.e., $H_{\infty}=\mathbb{P}^{2} \backslash \mathbb{A}^{2}$. For details, see [13] and the references therein. We warn, however, that there are $(X, f)$ for which the modified assertion does not hold true. (For example, consider a morphism $f=\left(f_{1}, f_{2}\right): \mathbb{P}^{1} \times \mathbb{P}^{1} \rightarrow \mathbb{P}^{1} \times \mathbb{P}^{1}$, where $f_{1}: \mathbb{P}^{1} \rightarrow \mathbb{P}^{1}$ is a linear map and $f_{2}: \mathbb{P}^{1} \rightarrow \mathbb{P}^{1}$ is a quadratic map.)

Let us go back to our setting where $f$ is an automorphism of a smooth projective surface $X$. Since the works of Gromov and Yomdin state that $\log \lambda$ is equal to the topological entropy $h_{\text {top }}\left(f_{\mathbb{C}}\right)$ of $f_{\mathbb{C}}$, the condition $\lambda>1$ in Assertion A means that $f_{\mathbb{C}}$ has positive topological entropy. It is known that, from a dynamical viewpoint, automorphisms of positive topological entropy enjoy many interesting properties (cf. $[7,8,9,10,18,19]$ ). According to Cantat $[7$, Proposition 1], if $X$ has an automorphism of positive topological entropy, then $X$ is either a non-minimal rational surface, or a surface birational to a $K 3$ surface, an Enriques surface or an abelian surface.

To single out $Z_{1}, Z_{2}$ and $Z_{3}$ in Assertion A, we first show the following. Here a curve means a reduced effective divisor on $X_{\mathbb{C}}$, and an irreducible curve $C$ is said to be $f_{\mathbb{C}}$-periodic if $f_{\mathbb{C}}^{n}(C)=C$ for some positive integer $n$.

Proposition B (cf. Proposition 3.1). Let $f_{\mathbb{C}}: X_{\mathbb{C}} \rightarrow X_{\mathbb{C}}$ be an automorphism of a smooth projective surface, which has positive topological entropy. Then there are only finitely many $f_{\mathbb{C}}$-periodic curves on $X_{\mathbb{C}}$.

In fact, we construct a nef and big $\mathbb{R}$-divisor class $\nu$ in the Néron-Severi group tensored

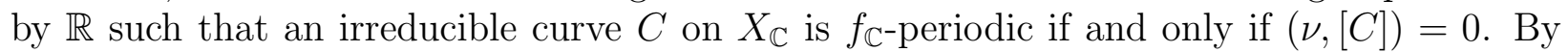

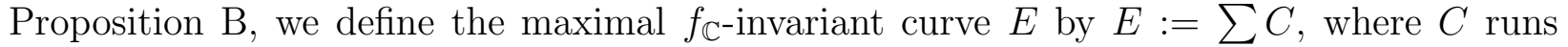

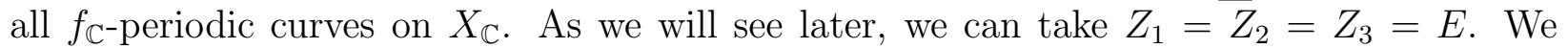
remark that $E=\emptyset$ if and only if $\nu$ is ample, and, in case $E \neq \emptyset$, general points on $E$ 
behaves differently from general points on $X \backslash E$ (Theorem 6.5). We also remark that, as a corollary of Proposition B, we see that an automorphism $f_{\mathbb{C}}$ of positive topological entropy has a Zariski-dense orbit (cf. Corollary 3.3 and [19, Theorem 1.4(2)]).

In the following, for simplicity, we say that $f$ has positive topological entropy if $f_{\mathbb{C}}$ does, and we use the notation $h_{\text {top }}(f)$ instead of $h_{\text {top }}\left(f_{\mathbb{C}}\right)$. We show in Lemma 3.8 that we can take an $\mathbb{R}$-divisor $D$ with $[D]=\nu$ such that $f^{*}(D)+f^{-1 *}(D)$ is $\mathbb{R}$-linearly equivalent to $\left(\lambda+\lambda^{-1}\right) D$. In our setting where $f: X \rightarrow X$ is defined over a number field $K \subset \mathbb{C}, E$ is defined over $\bar{K}$ and $D$ can be taken so that it is defined over $\bar{K}$ (Lemma 5.1 ).

The following is a precise form of Assertion $\mathrm{A}(1)$.

Theorem C (cf. Theorem 5.2). Let $X$ be a smooth projective surface over a number field $K \subset \mathbb{C}$, and $f: X \rightarrow X$ an automorphism of positive topological entropy $h_{\text {top }}(f)=\log \lambda$. Let $D$ be the nef and big $\mathbb{R}$-divisor as above, and $E$ the maximal $f$-invariant curve. Replacing $K$ by a suitable finite extension if necessary, we assume that $D$ and $E$ are defined over $K$. Then there exists a unique function

$$
\widehat{h}_{D}: X(\bar{K}) \rightarrow \mathbb{R}
$$

with the following two properties.

(i) $\widehat{h}_{D}$ is a height function corresponding to $D$.

(ii) $\widehat{h}_{D}(f(x))+\widehat{h}_{D}\left(f^{-1}(x)\right)=\left(\lambda+\lambda^{-1}\right) \widehat{h}_{D}(x)$ for all $x \in X(\bar{K})$.

Moreover, $\widehat{h}_{D}$ satisfies the following properties.

(iii) $\widehat{h}_{D}$ is non-negative.

(iv) For $x \in X(\bar{K}), \widehat{h}_{D}(x)=0$ if and only if $x$ lies on $E$ or $x$ is $f$-periodic.

(v) $\widehat{h}_{D}$ satisfies the Northcott finiteness property on $(X \backslash E)(\bar{K})$ (See Theorem 5.2 for a precise statement).

We call $\widehat{h}_{D}$ a canonical height function for $f$ and $D$.

In a pioneering paper [21], Silverman constructed canonical height functions $\widehat{h}$ on $K 3$ surfaces given by the complete intersection of $(1,1)$ and $(2,2)$ hypersurfaces in $\mathbb{P}^{2} \times \mathbb{P}^{2}$, and showed that $\widehat{h}$ enjoys properties comparable with the Néron-Tate height functions on abelian varieties. These $K 3$ surfaces have two involutions $\sigma_{1}, \sigma_{2}$ induced from the projections $p_{1}, p_{2}: X \rightarrow \mathbb{P}^{2}$. One sees that the composition $\sigma_{2} \circ \sigma_{1}$ has the positive topological entropy $\log (7+4 \sqrt{3})$, and $\widehat{h}$ is the canonical height function for $\sigma_{2} \circ \sigma_{1}$ and $(1+\sqrt{3})\left(p_{1}^{*} H+p_{2}^{*} H\right)$, where $H$ is a line in $\mathbb{P}^{2}$. From this view, Theorem $\mathrm{C}$ gives an extension of [21, Theorem 1.1] to any smooth projective surface automorphisms of positive topological entropy. We also note that Theorem C, together with Theorem D below, gives a refinement of $[14, \S 1]$ when $\left(X ; f, f^{-1}\right)$ is viewed as a dynamical eigensystem for $D$ of degree $\left(\lambda+\lambda^{-1}\right)$.

On the above $K 3$ surfaces, there are no $\left(\sigma_{2} \circ \sigma_{1}\right)$-periodic curves and so $D$ is ample. In general, projective surface automorphisms of positive topological entropy have non-empty $E$ and so $D$ cannot be ample. Still, there is an effective divisor $Z$ on $X$ such that $\operatorname{Supp}(Z) \subseteq$ $\operatorname{Supp}(E)$ and such that $D-\varepsilon Z$ is ample for sufficiently small $\varepsilon>0$. Moreover, since $E$ is a curve, the dynamical properties of $f$ restricted to $E$ are easy to understand. By these, together with Theorem C, we get Assertion A(2)(3). 
Theorem D (cf. Theorem 6.4, Corollary 6.6). With the notation and assumption in Theorem $C$, we have the following.

(1) The set $\{x \in(X \backslash E)(\bar{K}) \mid x$ is $f$-periodic $\}$ is of bounded height.

(2) Let $x \in(X \backslash E)(\bar{K})$ be a non $f$-periodic point. Then

$$
\lim _{T \rightarrow+\infty} \frac{N_{f, x}^{+}(T)}{\log T}=\frac{1}{\log \lambda}
$$

(Also see Theorem 6.5 for stronger statements.)

The organization of this paper is as follows. After reviewing some basic facts on smooth projective surfaces over $\mathbb{C}$ in $\S 1$, we construct a nef and big $\mathbb{R}$-divisor class $\nu$ in $\S 2$, with which we derive finiteness of $f$-periodic curves in $\S 3$. Also in $\S 3$, we give examples of automorphisms of positive topological entropy, and construct a nef and big $\mathbb{R}$-divisor $D$ with $[D]=\nu$. Now turning our attention to arithmetic properties, we briefly review on height functions in $\S 4$. In $\S 5$, we construct canonical height functions $\widehat{h}_{D}$ and study their further properties. In $\S 6$, we prove Theorem $\mathrm{D}$ and related results.

One approach to prove Theorems $\mathrm{C}$ and $\mathrm{D}$ would be to contract the curve $E$ on $X$ and to work over the singular surface $Y$ on which $E$ is contracted. In general, however, this is possible as analytic surfaces (cf. [11, Example 5.7.3]), and it is not clear to the author whether in our case $Y$ is algebraic (which is preferably $\mathbb{Q}$-factorial). So, we choose to work over $X$, using the ampleness of $D-\varepsilon Z$.

Cantat [7] showed the existence of the unique invariant probability measure $\mu$ of maximal entropy on $X$. Finally let us note that it would be an interesting question whether or not the Galois orbits of general periodic points (or of general points of small height) are asymptotically equidistributed with respect to $\mu$ (cf. [14, Question 3.4.1]).

Acknowledgments. I thank Professors Takeshi Abe, Vincent Maillot, Shigeru Mukai and Keiji Oguiso for valuable discussions and assistance. I thank the referee for carefully reading the manuscript and giving many helpful comments.

\section{Preliminaries on PRojeCtive SURFACES}

In this section, we review some facts on projective surfaces and fix notation that will be used in the sequel. We refer the reader to [2], [3] and [15] for details.

Let $X$ be a smooth projective surface over $\mathbb{C}$. A curve means a reduced effective divisor on $X$. We write for $\operatorname{Div}(X)$ the group of divisors on $X$, and $\operatorname{Cl}(X)$ for the divisor class group on $X$, i.e., the linearly equivalence classes of divisors on $X$. Let $N^{1}(X)$ be the Néron-Severi group, i.e., the numerically equivalence classes of divisors on $X$. We denote its rank by $\rho$. We write $\operatorname{Div}_{\mathbb{R}}(X):=\operatorname{Div}(X) \otimes \mathbb{R}$ for the $\mathbb{R}$-vector space of $\mathbb{R}$-divisors on $X$, and $\operatorname{Cl}(X)_{\mathbb{R}}$ for the $\mathbb{R}$-vector space of $\mathbb{R}$-linearly equivalence classes of $\mathbb{R}$-divisors on $X$, and $N^{1}(X)_{\mathbb{R}}$ for the $\mathbb{R}$-vector space of numerically equivalence classes of $\mathbb{R}$-divisors on $X$. We note that $\mathrm{Cl}(X)_{\mathbb{R}}=\mathrm{Cl}(X) \otimes \mathbb{R}$ and $N^{1}(X)_{\mathbb{R}}=N^{1}(X) \otimes \mathbb{R}$. For $\mathbb{R}$-divisors $D_{1}, D_{2}$, we write $D_{1} \sim_{\mathbb{R}} D_{2}$ if $D_{1}, D_{2}$ are $\mathbb{R}$-linearly equivalent. For an $\mathbb{R}$-divisor $D$, we denote by $[D]$ its image in $N^{1}(X)_{\mathbb{R}}$. An $\mathbb{R}$-divisor $D$ is said to be integral if it lies in the image of the natural map $\operatorname{Div}(X) \rightarrow \operatorname{Div}(X)_{\mathbb{R}}$ 
An $\mathbb{R}$-divisor $D$ is said to be ample (resp. big, effective) if $D$ is expressed as a finite sum $D=\sum c_{i} D_{i}$ for some $c_{i}>0$ and ample (resp. big, effective) integral divisors $D_{i}$. An $\mathbb{R}$-divisor $D$ is said to be nef if $(D, C) \geq 0$ for all irreducible curves $C$ on $X$. Amplitude, bigness and nefness depend only on numerically equivalence classes. Let $\operatorname{Amp}(X) \subset N^{1}(X)_{\mathbb{R}}$ and $\operatorname{Nef}(X) \subset N^{1}(X)_{\mathbb{R}}$ denote the ample cone and the nef cone in $N^{1}(X)_{\mathbb{R}}$, respectively. Kleiman's theorem states that the closure of the ample cone is equal to the nef cone ([15, Theorem 1.4.23]):

$$
\overline{\operatorname{Amp}(X)}=\operatorname{Nef}(X)
$$

We collect some facts on $\mathbb{R}$-divisors. For a proof, see for example [15, Proposition 2.2.22, the proof of Theorem 2.3.18].

Proposition 1.1. Let $D$ be an $\mathbb{R}$-divisor on $X$.

(1) $D$ is big if and only if $[D]=[A]+[N]$ in $N^{1}(X)_{\mathbb{R}}$, where $A$ is an ample $\mathbb{R}$-divisor and $N$ is an effective $\mathbb{R}$-divisor.

(2) Assume $D$ is nef. Then $D$ is big if and only if $\left(D^{2}\right)>0$.

Since $N^{1}(X)_{\mathbb{R}}$ has signature $(1, \rho-1)$ by the Hodge index theorem, the set $\left\{x \in N^{1}(X)_{\mathbb{R}} \mid\right.$ $(x, x)>0\}$ consists of two disjoint cones. Let $P$ be the positive cone, i.e., one of the cones that contains an ample divisor. Let $\bar{P}$ be the closure of $P$ in $N^{1}(X)_{\mathbb{R}}$. The following elementary lemma will be useful. See for example [2, IV, Cor. 7.2] about (1) and (2). The Cauchy-Schwartz inequality yields (3).

Lemma 1.2. (1) If $x, y \in \bar{P}$, then $(x, y) \geq 0$.

(2) If $x \in \bar{P} \backslash\{0\}$ and $y \in P$, then $(x, y)>0$.

(3) If $x, y \in \bar{P} \backslash\{0\}$ and $x \neq$ ty for all $t \in \mathbb{R}_{>0}$, then $(x, y)>0$.

The next proposition will be used in $\S 3$ and $\S 5$.

Proposition 1.3. Let $\nu \in N^{1}(X)_{\mathbb{R}}$ be a nef and big $\mathbb{R}$-divisor class on $X$. We set

$$
Z(\nu):=\{C \mid C \text { is an irreducible curve on } X \text { such that }(\nu,[C])=0\} \text {. }
$$

Then

(1) $Z(\nu)$ is a finite set.

(2) There is an effective divisor $Z$ on $X$ such that $\operatorname{Supp}(Z) \subseteq \bigcup_{C \in Z(\nu)} \operatorname{Supp}(C)$ and such that $\nu-\varepsilon[Z]$ is ample for all sufficiently small $\varepsilon>0$.

Proof. (1) From Proposition 1.1, there are an ample $\mathbb{R}$-divisor $A$ and an effective $\mathbb{R}$ divisor $N$ such that $\nu=[A]+[N]$. If an irreducible curve $C$ satisfies $(\nu,[C])=0$, then $(N, C)=-(A, C)<0$. Hence $C$ must be an irreducible component of $\operatorname{Supp}(N)$. Since the number of irreducible components of $\operatorname{Supp}(N)$ is finite, $Z(\nu)$ is a finite set.

(2) We set $Z(\nu)=\left\{C_{1}, \ldots, C_{m}\right\}$. Since $\left(\nu^{2}\right)>0$, the Hodge index theorem asserts that the intersection matrix $\left(\left(C_{i}, C_{j}\right)\right)_{i, j} \leq 0$. Then there is an effective divisor $Z_{1}=\sum_{i=1}^{m} a_{i} C_{i}$ such that, for all $i,\left(Z_{1}, C_{i}\right) \leq 0$ and $a_{i}>0$ (cf. [2, Chap. I (2.10)]).

We claim that $\nu-\varepsilon\left[Z_{1}\right]$ is nef and big for all sufficiently small $\varepsilon>0$. Indeed, since $\left(\nu^{2}\right)>0$, we have $\left(\left(\nu-\varepsilon\left[Z_{1}\right]\right)^{2}\right)>0$ for $1 \gg \varepsilon>0$. Let $C$ be an irreducible curve on $X$. If $C \in Z(\nu)$, then $\left(\nu-\varepsilon\left[Z_{1}\right],[C]\right)=-\varepsilon\left(Z_{1}, C\right) \geq 0$. If $C \subseteq \operatorname{Supp}(N)$ but $C \notin Z(\nu)$, then $(\nu,[C])>0$, so 
that $\left(\nu-\varepsilon\left[Z_{1}\right],[C]\right)>0$ for $1 \gg \varepsilon>0$, On the other hand, since $Z(\nu) \subseteq \operatorname{Supp}(N), N-\varepsilon Z_{1}$ is effective for $1 \gg \varepsilon>0$ and $\operatorname{Supp}\left(N-\varepsilon Z_{1}\right) \subseteq \operatorname{Supp}(N)$. Thus, if $C \nsubseteq \operatorname{Supp}(N)$, then $\left(\nu-\varepsilon\left[Z_{1}\right],[C]\right)>\left(N-\varepsilon Z_{1}, C\right) \geq 0$. We conclude that $\nu-\varepsilon\left[Z_{1}\right]$ is nef and big for $1 \gg \varepsilon>0$. There is some $C_{i}$ with $\left(Z_{1}, C_{i}\right)<0$. (Indeed, if $\left(Z_{1}, C_{i}\right)=0$ for all $i$, then $Z_{1}=\sum_{i=1}^{m} a_{i} C_{i}$ is numerically trivial by the Hodge index theorem, which is absurd because $a_{i}>0$ for all $i$.) Hence

$$
Z\left(\nu-\varepsilon\left[Z_{1}\right]\right):=\left\{C \mid C \text { is an irreducible curve on } X \text { such that }\left(\nu-\varepsilon\left[Z_{1}\right],[C]\right)=0\right\}
$$

is a proper subset of $Z(\nu)$. Inductively, we apply the above argument to $\nu-\varepsilon\left[Z_{1}\right]$, we see that there is an effective divisor $Z$ on $X$ with $\operatorname{Supp}(Z) \subseteq \bigcup_{C \in Z(\nu)} \operatorname{Supp}(C)$ such that $\nu-\varepsilon[Z]$ is nef and big for $1 \gg \varepsilon>0$ and such that

$$
Z(\nu-\varepsilon[Z]):=\{C \mid C \text { is an irreducible curve on } X \text { such that }(\nu-\varepsilon[Z],[C])=0\}
$$

is empty. Then the Nakai criterion for $\mathbb{R}$-divisors due to Campana and Peternell ([15, Theorem 2.3.18]) yields that $\nu-\varepsilon[Z]$ is ample for $1 \gg \varepsilon>0$.

\section{NeF AND BIG $\mathbb{R}$-DIVISOR CLASSES}

Let $X$ be a smooth projective surface over $\mathbb{C}$, and $f: X \rightarrow X$ an automorphism. Then $f$ induces $f^{*}: H^{2}(X, \mathbb{Z}) \rightarrow H^{2}(X, \mathbb{Z})$. By a slight abuse of notation, we also denote by $f^{*}$ the induced transformations on $H^{2}(X, \mathbb{R})$ and on $H^{2}(X, \mathbb{C})$. Let

$$
\lambda:=\sup \left\{|t| \mid \operatorname{det}\left(t I-f^{*}\right)=0\right\}
$$

be the spectral radius of $f^{*}$.

By the works of Gromov and Yomdin, the topological entropy of $f$ is related to the spectral radius of $f^{*}$, which we might take as one of the definitions of topological entropy. We put together in Theorem 2.1 the works of Gromov and Yomdin and other results which will be needed in the sequel. For Theorem 2.1(1), we refer the reader to [8, Theorem 2.1.5] and [10, Théorème 2.1]. For Theorem 2.1(2), we refer the reader to [18, Theorem 3.2]. We note that $K 3$ surfaces are treated in [18, Theorem 3.2], but since the signature of $H^{1,1}(X, \mathbb{R})$ is $(1, \rho-1)$, the argument there also applies to automorphisms of smooth projective surfaces.

Theorem 2.1 (cf. $[8,10,18]$ ). Let $f: X \rightarrow X$ be an automorphism of a smooth projective surface on $X$.

(1) The topological entropy $h_{\mathrm{top}}(f)$ is equal to $\log \lambda$. Moreover, $h_{\mathrm{top}}(f)$ is also equal to the spectral radius of $\left.f^{*}\right|_{H^{1,1}(X, \mathbb{R})}$ acting on $H^{1,1}(X, \mathbb{R})$.

(2) Assume $f$ has positive topological entropy, i.e., $\lambda>1$. Then the set of eigenvalues of $f^{*}$ with counted multiplicities is given by

$$
\left\{\lambda, \lambda^{-1}, \alpha_{1}, \alpha_{2}, \cdots, \alpha_{\operatorname{dim} H^{2}(X, \mathbb{R})-2}\right\},
$$

where $\left|\alpha_{i}\right|=1$ for all $i=1,2, \ldots, \operatorname{dim} H^{2}(X, \mathbb{R})-2$. In particular, $f^{*}$ has a unique, simple eigenvalue $\beta$ with $|\beta|>1$, and $\beta$ is equal to $\lambda$.

Lemma 2.2. Let $X$ be a smooth projective surface over $\mathbb{C}$, and $f: X \rightarrow X$ an automorphism. We assume that the Kodaira dimension of $X$ is non-negative. Let $X_{0}$ be the minimal surface of $X$, and $\pi: X \rightarrow X_{0}$ the contraction morphism. Then there is a unique automorphism $f_{0}: X_{0} \rightarrow X_{0}$ with $\pi \circ f=f_{0} \circ \pi$. Moreover, $h_{\text {top }}\left(f_{0}\right)=h_{\text {top }}(f)$. 
Proof. The automorphism $f: X \rightarrow X$ induces a birational map $f_{0}: X_{0}-\rightarrow X_{0}$ with $\pi \circ f=f_{0} \circ \pi$. Since the Kodaira dimension of $X$ is non-negative, $f_{0}$ is an automorphism $([3$, Theorem V.19]).

Let $\left\{F_{1}, \ldots, F_{k}\right\}$ be the set of exceptional irreducible curves of $\pi$, i.e., the set of irreducible curves $F_{i}$ 's that are contracted to points by $\pi$. Then $H^{2}(X, \mathbb{Z})=\pi^{*}\left(H^{2}\left(X_{0}, \mathbb{Z}\right)\right) \oplus \bigoplus_{i=1}^{k} \mathbb{Z}\left[F_{i}\right]$.

Since $\pi \circ f=f_{0} \circ \pi, f^{*}$ preserves $\pi^{*}\left(H^{2}\left(X_{0}, \mathbb{Z}\right)\right)$ and $\bigoplus_{i=1}^{k} \mathbb{Z}\left[F_{i}\right]$. On the other hand, since the intersection form on $\bigoplus_{i=1}^{k} \mathbb{Z}\left[F_{i}\right]$ is negative definite, Lemma 2.3 below asserts that the eigenvalues of $\left.f^{*}\right|_{\bigoplus_{i=1}^{k} \mathbb{Z}\left[F_{i}\right]}$ lie on the unit circle. Hence $f_{0}$ has the same topological entropy as $f$ by Theorem 2.1(1).

Lemma 2.3 ([18], Lemma 3.1). Let $O(p, q) \subset \mathrm{GL}_{p+q}(\mathbb{R})$ be the orthogonal group of the real quadratic form $x_{1}^{2}+\cdots+x_{p}^{2}-x_{p+1}^{2}-\cdots-x_{p+q}^{2}$ of signature $(p, q)$. Then, for any $T \in O(p, q)$, $T$ has at most $\min (p, q)$ eigenvalues outside the unit circle, counted with multiplicities.

Cantat [7, Proposition 1] classified smooth projective surfaces that have automorphisms of positive topological entropy (cf. Lemma 2.2).

Proposition 2.4 ([7], Proposition 1). Let $X$ be a smooth projective surface over $\mathbb{C}$ which has an automorphism of positive topological entropy. Then $X$ is either a non-minimal rational surface, or a surface birational to a $K 3$ surface, an Enriques surface or an abelian surface.

From now on, we consider automorphisms of positive topological entropy. The next proposition concerns the existence of a nef and big $\mathbb{R}$-divisor class $\nu$ that transforms well relative to $f$. This may be seen as an $\mathbb{R}$-divisor class version of [7, Théorème 2] and [9, Theorem 5.1].

Proposition 2.5. Let $X$ be a smooth projective surface over $\mathbb{C}$, and $f: X \rightarrow X$ an automorphism of positive topological entropy $\log \lambda$.

(1) There are non-zero nef classes $\nu_{+}$and $\nu_{-} \in \operatorname{Nef}(X)$ such that $f^{*}\left(\nu_{+}\right)=\lambda \nu_{+}$and $f^{*}\left(\nu_{-}\right)=\lambda^{-1} \nu_{-}$.

(2) We have $\left(\nu_{+}^{2}\right)=0$ and $\left(\nu_{-}^{2}\right)=0$. If $\nu_{+}^{\prime}, \nu_{-}^{\prime} \in \operatorname{Nef}(X)$ are other nef classes with $f^{*}\left(\nu_{+}^{\prime}\right)=\lambda \nu_{+}^{\prime}, f^{*}\left(\nu_{-}^{\prime}\right)=\lambda^{-1} \nu_{-}^{\prime}$, then $\nu_{+}^{\prime}=c_{+} \nu_{+}, \nu_{-}^{\prime}=c_{-} \nu_{-}$for some $c_{+}, c_{-}>0$.

(3) Set $\nu:=\nu_{+}+\nu_{-} \in N^{1}(X)_{\mathbb{R}}$. Then $\nu$ is nef and big. Moreover, one has

$$
f^{*}(\nu)+f^{-1 *}(\nu)=\left(\lambda+\lambda^{-1}\right) \nu .
$$

Proof. (1)(2) This is remarked in [8, Remerque 1.1] for $K 3$ surfaces, whose argument also applies to other surfaces. For the sake of completeness, we briefly give a proof.

By Theorem 2.1(2), there are non-zero $\nu_{+}$and $\nu_{-} \in H^{2}(X, \mathbb{R})$ such that $f^{*}\left(\nu_{+}\right)=\lambda \nu_{+}$ and $f^{*}\left(\nu_{-}\right)=\lambda^{-1} \nu_{-}$. We claim that the classes $\nu_{+}$and $\nu_{-}$belong to $N^{1}(X)_{\mathbb{R}}$. Indeed, we set

$$
W:=\left\{x \in H^{2}(X, \mathbb{R}) \mid(x, \delta)=0 \text { for all } \delta \in N^{1}(X)\right\} .
$$

If $X$ is a rational surface or a surface birational to an Enriques surface, then $W=0$, so that we get the claim trivially. If $X$ is a surface birational to an $K 3$ surface or an abelian surface, then the Lefschetz $(1,1)$-theorem tells us that $\left.f^{*}\right|_{W}$ is conjugate to an element of $O(2,0) \times O\left(0, \operatorname{dim} H^{2}(X, \mathbb{R})-2-\rho\right)$. Then, from Lemma 2.3, all eigenvalues of $\left.f^{*}\right|_{W}$ lie on the unit circle. Thus we get the claim in this case also. 
Now Proposition 2.5(1)(2) is proven as in [7, Théorème 2] and [9, Theorem 5.1]. Noting that $\operatorname{Amp}(X)$ spans $N^{1}(X)_{\mathbb{R}}$ as an $\mathbb{R}$-vector space, we take a general element $\gamma$ in $\operatorname{Amp}(X)$ and fix it. Since $\lambda$ is the unique, simple eigenvalue of $f^{*}$ whose absolute value is greater than 1 by Theorem 2.1(2), $\frac{1}{\lambda^{n}}\left(f^{*}\right)^{n}(\gamma)$ converges to $c \nu_{+}$with some $c \neq 0$ as $n \rightarrow+\infty$. We replace $\nu_{+}$by $c \nu_{+}$. Since $\frac{1}{\lambda^{n}}\left(f^{*}\right)^{n}(\gamma) \in \operatorname{Amp}(X)$ for all $n \geq 1$, we see that $\nu_{+} \in \operatorname{Nef}(X)$ by Kleiman's theorem (1.1). A similar argument shows that $\nu_{-}$can also be taken in $\operatorname{Nef}(X)$.

It follows from $\lambda^{2}\left(\nu_{+}^{2}\right)=\left(f^{*}\left(\nu_{+}\right)^{2}\right)=\left(\nu_{+}^{2}\right)$ that $\left(\nu_{+}^{2}\right)=0$. Similarly, $\left(\nu_{-}^{2}\right)=0$. By Theorem 2.1(2), $\nu_{+}$is unique up to positive real numbers and so is $\nu_{-}$.

(3) Obviously, $\nu=\nu_{+}+\nu_{-}$is nef. Since $\left(\nu^{2}\right)=\left(\nu_{+}^{2}\right)+2\left(\nu_{+}, \nu_{-}\right)+\left(\nu_{-}^{2}\right)=2\left(\nu_{+}, \nu_{-}\right)$, Lemma 1.2(3) yields that $\left(\nu^{2}\right)>0$. By Proposition 1.1(2), $\nu$ is big. We have

$$
\begin{aligned}
f^{*}(\nu)+f^{-1 *}(\nu) & =f^{*}\left(\nu_{+}\right)+f^{*}\left(\nu_{-}\right)+f^{-1 *}\left(\nu_{+}\right)+f^{-1 *}\left(\nu_{-}\right) \\
& =\left(\lambda+\lambda^{-1}\right)\left(\nu_{+}+\nu_{-}\right)=\left(\lambda+\lambda^{-1}\right) \nu .
\end{aligned}
$$

Remark 2.6. If $\lambda$ is a quadratic irrational number, then $\nu$ can be taken as an integral divisor class, i.e., $\nu \in N^{1}(X)$. Indeed, in this case, $\lambda$ is a root of the quadratic polynomial $t^{2}-\tau t+1$, where $\tau:=\lambda+\lambda^{-1}$ is an integer. We set $F=\mathbb{Q}(\lambda)$ and let $O_{F}$ be the ring of integers of $F$. Let $\sigma \in \operatorname{Gal}(F / \mathbb{Q})$ be the non-trivial element.

Then there is a nef class $\nu_{+} \in N^{1}(X) \otimes_{\mathbb{Z}} O_{F}$ with $f^{*} \nu_{+}=\lambda \nu_{+}$. We set $\nu_{-}:=\sigma\left(\nu_{+}\right) \in$ $N^{1}(X) \otimes_{\mathbb{Z}} O_{F}$. Then $\nu_{-}$is nef and $f^{*}\left(\nu_{-}\right)=\lambda^{-1} \nu_{-}$. We set $\nu:=\nu_{+}+\nu_{-}$. Since $\sigma(\nu)=\nu$, we see that $\nu \in N^{1}(X)$.

\section{Finiteness for $f$-Periodic CURVES}

Let $X$ be a smooth projective surface over $\mathbb{C}$, and $f: X \rightarrow X$ an automorphism of positive topological entropy. A point $x$ on $X$ is said to be $f$-periodic if $f^{n}(x)=x$ for some positive integer $n$. An irreducible curve $C$ on $X$ is said to be $f$-periodic if $f^{n}(C)=C$ for some positive integer $n$. Note that an $f$-periodic $C$ may not be fixed pointwisely by $f$.

By [7, Théorème in p. 906], if $f$ has positive topological entropy, then there are infinitely many $f$-periodic points on $X$. Indeed, suppose there are only finitely many periodic points, then the support of the measure of $\mu$ in op.cit. is a finite set. This is impossible since the measure entropy with respect to $\mu$ is $\log \lambda>0$.

In contrast, the next proposition shows that there are only finitely many $f$-periodic curves on $X$.

Proposition 3.1. Let $X$ be a smooth projective surface over $\mathbb{C}$, and $f: X \rightarrow X$ an automorphism of positive topological entropy.

(1) There are only finitely many $f$-periodic curves on $X$. If $X$ is non-rational and $C$ is an $f$-periodic curve, then $C$ is isomorphic to $\mathbb{P}^{1}$.

(2) Let $\nu \in N^{1}(X)_{\mathbb{R}}$ be a nef and big class in Proposition 2.5. Then an irreducible curve $C$ is $f$-periodic if and only if $([C], \nu)=0$. Moreover, if $X$ is non-rational and $C$ is an irreducible curve with $([C], \nu)=0$, then $C$ is isomorphic to $\mathbb{P}^{1}$. 
Proof. In virtue of Proposition 1.3, we have only to show (2). Let $\log \lambda$ denote the topological entropy of $f$.

Suppose $C$ is an $f$-periodic curve, i.e., $f^{n}(C)=C$ for some positive integer $n$. For $k \in \mathbb{Z}$, we then have

$$
\begin{aligned}
\left([C], \nu_{+}\right)+\left([C], \nu_{-}\right) & =([C], \nu) \\
& =\left(\left[f^{k n}(C)\right], \nu\right)=\left([C],\left(f^{*}\right)^{k n}(\nu)\right) \\
& =\left([C],\left(f^{*}\right)^{k n}\left(\nu_{+}\right)\right)+\left([C],\left(f^{*}\right)^{k n}\left(\nu_{-}\right)\right) \\
& =\lambda^{k n}\left([C], \nu_{+}\right)+\lambda^{-k n}\left([C], \nu_{-}\right) .
\end{aligned}
$$

Since Eqn. (3.1) holds for all $k \in \mathbb{Z}$, we obtain $\left([C], \nu_{+}\right)=0$ and $\left([C], \nu_{-}\right)=0$, whence $([C], \nu)=0$.

Next, suppose $C$ is an irreducible curve with $([C], \nu)=0$. Since $\nu_{+}$and $\nu_{-}$are nef classes, we get $\left([C], \nu_{+}\right)=0$ and $\left([C], \nu_{-}\right)=0$. Then

$$
\left(\left[f^{k}(C)\right], \nu\right)=\lambda^{k}\left([C], \nu_{+}\right)+\lambda^{-k}\left([C], \nu_{-}\right)=0
$$

for all $k \in \mathbb{Z}$. Proposition 1.3(2) yields that $f^{k}(C)=f^{l}(C)$ for some positive integers $k<l$. Thus $f^{l-k}(C)=C$.

Finally, suppose that $X$ is non-rational. By Proposition 2.4, $X$ is birational to a $K 3$ surface, an Enriques surface, or an abelian surface. Let $C$ be an $f$-periodic curve.

Case 1: The case $X$ is minimal. Since $\left(\nu^{2}\right)>0$ and $(\nu,[C])=0$, the Hodge index theorem yields that $\left(C^{2}\right)<0$. Since $K_{X}$ is numerically trivial, we have $\left(C, K_{X}\right)=0$. Then the adjunction formula shows that $C$ is a smooth rational curve with selfintersection -2 .

Case 2: The case $X$ is not minimal. Noting that $X$ is non-rational, let $X_{0}$ be the minimal surface of $X$, and $\pi: X \rightarrow X_{0}$ the contraction morphism. By Lemma 2.2, $f$ induces the automorphism $f_{0}: X_{0} \rightarrow X_{0}$ with $h_{\text {top }}\left(f_{0}\right)=\log \lambda$.

If $C$ is contracted to a point by $\pi$, then $C$ is isomorphic to $\mathbb{P}^{1}$. If $C$ is not contracted to a point by $\pi$, then we set $C_{0}=\pi(C)$. Since $\pi \circ f=f_{0} \circ \pi, C_{0}$ is $f_{0}$-periodic. Then, by Case $1, C_{0}$ is isomorphic to $\mathbb{P}^{1}$. Since $\pi$ is a succession of blow-ups, $\left.\pi\right|_{C}: C \rightarrow C_{0}$ is birational. Hence $C$ is isomorphic to $\mathbb{P}^{1}$.

A curve $D$ on $X$ is said to be $f$-invariant if $f(D)=D$. We define the curve $E$ by

$$
E=\sum C
$$

where $C$ runs all $f$-periodic curves. Then $E$ is $f$-invariant. We will call $E$ the maximal $f$-invariant curve. This terminology may be justified from the following proposition.

Proposition 3.2. The curve $E$ is maximal among $f$-invariant curves. Namely, if $D$ is an $f$-invariant curve, then $E-D$ is effective.

Proof. Let $D=\sum_{i=1}^{k} D_{i}$ be the irreducible decomposition of $D$. Then $f$ acts on the set $\left\{D_{1}, \ldots, D_{k}\right\}$ as a permutation. In particular, $f^{k !}\left(D_{i}\right)=D_{i}$ for all $i$. This shows that $D_{i}$ 's are $f$-periodic, and thus $E-D$ is effective.

The following corollary gives a different proof of [19, Theorem 1.4(2)] (cf. Remark 3.4), when $X$ is an algebraic $K 3$ surface. 
Corollary 3.3. Let $X$ be a smooth projective surface over $\mathbb{C}$, and $f: X \rightarrow X$ an automorphism of positive topological entropy. If $x$ is not $f$-periodic and does not lie on $E$, then the orbit $\left\{f^{n}(x) \mid n \in \mathbb{Z}\right\}$ is Zariski dense in $X$.

Proof. Let $W$ be the Zariski closure of $\left\{f^{n}(x) \mid n \in \mathbb{Z}\right\}$. To derive a contradiction, we assume $W \neq X$. Since the one dimensional component $W_{1}$ of $W$ satisfies $f\left(W_{1}\right)=W_{1}$, we have $W_{1} \subseteq \operatorname{Supp}(E)$ by Proposition 3.2. Since $x$ does not lie on $E$, we conclude $W_{1}=\emptyset$. Then $W$ becomes a finite set, which contradicts with the assumption that $x$ is not $f$-periodic.

Remark 3.4. In contrast, suppose $X$ is a $K 3$ surface and $f: X \rightarrow X$ is an automorphism of null topological entropy. Then $X=\bigcup C$ as a set, where $C$ runs all $f$-periodic curves. Indeed, by [8, Proposition 1.4, Corollaire 2.2], either $f^{n}=$ id for some positive integer $n$, or there is a surjective morphism $\pi: X \rightarrow \mathbb{P}^{1}$ with $\pi \circ f=\pi$. In the first case, every curve is $f$-periodic. In the second case, every curve in a fiber is $f$-periodic.

For illustration, we give some examples of projective surface automorphisms of positive topological entropy. For other examples, we refer the reader to [8] and [18]. See also [14, $\S 1.4]$ and the references therein.

Example 3.5 ( $K 3$ surfaces in $\mathbb{P}^{2} \times \mathbb{P}^{2}$ ). Let $X$ be a $K 3$ surface in $\mathbb{P}^{2} \times \mathbb{P}^{2}$ given by the intersection of hypersurfaces of bidegree $(1,1)$ and $(2,2)$. This surface is sometimes called a Wehler K3 surface (cf. [17]). When $X$ is defined over a number field $K$, Silverman [21] constructed a canonical height function on $X(\bar{K})$ and deduced arithmetic properties of $X$.

For $i=1,2$, we write $p_{i}: X \rightarrow \mathbb{P}^{2}$ for the projection to the $i$-th factor, and $\sigma_{i}: X \rightarrow X$ the involution given by $p_{i}$. We take the composition of these two involutions:

$$
f=\sigma_{2} \circ \sigma_{1} \text {. }
$$

Let $H$ be a hyperplane in $\mathbb{P}^{2}$, and we set $H_{i}=p_{i}^{-1}(H)$. As in $[21, \S 1]$, we put

$$
\nu_{+}=(2+\sqrt{3})\left[H_{1}\right]-\left[H_{2}\right], \quad \nu_{+}=-\left[H_{1}\right]+(2+\sqrt{3})\left[H_{2}\right]
$$

in $N^{1}(X)_{\mathbb{R}}$. By $\left[21\right.$, Lemma 2.1], $\nu_{+}, \nu_{-}$are nef and satisfy

$$
f^{*}\left(\nu_{+}\right)=(7+4 \sqrt{3}) \nu_{+}, \quad f^{*}\left(\nu_{-}\right)=(7+4 \sqrt{3})^{-1} \nu_{-} .
$$

The morphism $f$ has the positive topological entropy $\log (7+4 \sqrt{3})$. Here $\nu:=\nu_{+}+\nu_{-}=(1+$ $\sqrt{3})\left(\left[H_{1}\right]+\left[H_{2}\right]\right)$ is ample, and there are no $f$-periodic curves on $X$ (cf. Proposition 3.1(2)).

Another example of a $K 3$ surface is a smooth hypersurface of tridegree $(2,2,2)$ in $\mathbb{P}^{1} \times \mathbb{P}^{1} \times$ $\mathbb{P}^{1}$. It has three natural involutions $\sigma_{1}, \sigma_{2}, \sigma_{3}$, and the composition $f:=\sigma_{3} \circ \sigma_{2} \circ \sigma_{1}$ has the positive topological entropy $\log (9+4 \sqrt{5})$. In this case also, there are no $f$-periodic curves. we refer the reader to $[1,17,22]$, for its arithmetical properties. See also [18, Introduction].

Example 3.6 (More K3 surface automorphisms). Let $S$ be an even lattice of signature $(1,1)$, which represents neither 0 nor -2 . Then $O(S):=\{g: S \rightarrow S \mid g$ is a lattice isometry $\}$ is infinite, and there is $g_{S}$ such that the eigenvalues of $g$ are given by $\lambda, \lambda^{-1}$ for some $\lambda>1$ (cf. [20, Example in p. 581]). 
Let $L_{K 3}$ denotes the $K 3$ lattice, i.e., the even unimodular lattice of signature $(3,19)$. We take a primitive embedding $S \hookrightarrow L_{K 3}$ ([2, Chap. I, Theorem (2.9)]), by which we regard $S$ as a sublattice of $L_{K 3}$. Let $T$ be an orthogonal complement of $S$ in $L_{K 3}$. We set $g:=\left(g_{S}, 1_{T}\right)$ acting on $S \oplus T$. Since $S^{*} / S$ is a finite group, by replacing $g$ by $g^{n}$ for some positive integer $n$, we may assume that $g$ acts on $S^{*} / S$ trivially. Then $g$ extends to a lattice isometry $g: L_{K 3} \rightarrow L_{K 3}$.

We take a $K 3$ surface $X$ and an isometry $\rho: H^{2}(X, \mathbb{Z}) \rightarrow L_{K 3}$ such that the Néron-Severi lattice and the transcendental lattice of $X$ are respectively given by $S$ and $T$ via $\rho$. Since $g$ acts on $T$ identically, $g$ is a Hodge isometry. Moreover, since $S$ does not represent $-2, g$ preserves the ample cone. Then Torelli's theorem ([2, Chap. VIII, Proposition (6.2)]) yields that there is an automorphism $f: X \rightarrow X$ such that $f^{*}$ is conjugate to $g$ via $\rho$. The automorphism $f$ has topological entropy $\log \lambda>0$.

Example 3.7 (Abelian surfaces and Kummer surfaces). Following [8] and [18, §4], we present some automorphisms of abelian surfaces and Kummer surfaces of positive topological entropy. (Some of the notation here will be used in the proof of Lemma 3.8.)

Let $X$ be an abelian surface over $\mathbb{C}$, and $f: X \rightarrow X$ an automorphism. We fix an analytic isomorphism $X \simeq \mathbb{C}^{2} / \Lambda$, where $\Lambda$ is a lattice in $\mathbb{C}^{2}$. We put $v:=f(0)$, and let $t_{v}: X \rightarrow X$ the translation by $v$. Then there is a group isomorphism $g: X \rightarrow X$ such that $f=t_{v} \circ g$.

Let $V \in \mathbb{C}^{2}$ be a lift of $v$, and $T_{V}: \mathbb{C}^{2} \rightarrow \mathbb{C}^{2}$ the translation by $V$. We denote by $G: \mathbb{C}^{2} \rightarrow \mathbb{C}^{2}$ the lift of $g$ such that $G(0)=0$. Then $F:=T_{V} \circ G$ is the lift of $f$ such that $F(0)=V$. We take the matrix $P=\left(\begin{array}{ll}p & q \\ r & s\end{array}\right) \in \mathrm{GL}_{2}(\mathbb{C})$ representing $G$. Let $\alpha, \beta$ be the roots of the characteristic polynomial of $P$ such that $|\alpha| \geq|\beta|$.

By identifying $H^{1,1}(X, \mathbb{R})$ as the space of translation-invariant $(1,1)$-forms on $X$, we compute the action of $f^{*}$ on $H^{1,1}(X, \mathbb{R})$ to find that the spectral radius $\lambda$ of $\left.f^{*}\right|_{H^{1,1}(X, \mathbb{R})}$ is given by $\lambda=|\alpha|^{2}$ (cf. [18, §4]). We also note that $|\alpha \beta|=|p s-q r|=1$ (cf. [18, §4]). In particular, if $|p+s|>2$, then $f$ has positive topological entropy.

Let $[-1]: X \rightarrow X$ be the inverse morphism. Let $K$ be the Kummer surface associated with $X$, i.e., the $K 3$ surface obtained by resolving the 16 ordinary double points of $X /[-1]$. In the sequel, we assume $v=0$, i.e., $f=g$. In this case, since $f$ commutes with $[-1], f$ induces the isomorphism $\bar{f}: K \rightarrow K$. By [18, §4], $\bar{f}$ has the same topological entropy as $f$.

Let $C$ be a nodal curve on $K$ that corresponds to one of the 16 ordinary double points of $X /[-1]$. Then $C$ is $\bar{f}$-periodic. Specifically, if $C_{0}$ be the nodal curve which corresponds to the origin of $X /[-1]$, then $\bar{f}\left(C_{0}\right)=C_{0}$ and $\left.\bar{f}\right|_{C_{0}}: C_{0} \rightarrow C_{0}$ is conjugate to

$$
\mathbb{P}^{1} \ni\left(x_{0}: x_{1}\right) \mapsto\left(p x_{0}+q x_{1}: r x_{0}+s x_{1}\right) \in \mathbb{P}^{1}
$$

via a suitable isomorphism $C \cong \mathbb{P}^{1}$.

For concrete examples, we consider an abelian surface $X$ which is the product of an elliptic curve $E$ and itself: $X=E \times E$. Then for each $P=\left(\begin{array}{ll}p & q \\ r & s\end{array}\right) \in \mathrm{SL}_{2}(\mathbb{Z})$ with $|p+s|>2$, the group isomorphism $f: X \rightarrow X$ given by $f(x, y)=(p x+q y, r x+s y)$ induces the automorphism $\bar{f}: K \rightarrow K$ of positive topological entropy.

In the rest of this section, we consider a version of Proposition 2.5 for $\mathbb{R}$-divisors modulo $\mathbb{R}$-linearly equivalence class. 
Lemma 3.8. Let the notation and assumption be as in Proposition 2.5.

(1) Then there are $\mathbb{R}$-divisors $D_{+}$and $D_{-}$on $X$ with $\left[D_{+}\right]=\nu_{+}$and $\left[D_{-}\right]=\nu_{-}$that satisfy

$$
f^{*}\left(D_{+}\right) \sim_{\mathbb{R}} \lambda D_{+} \quad \text { and } f^{*}\left(D_{-}\right) \sim_{\mathbb{R}} \lambda^{-1} D_{-} .
$$

Further, if $X$ is not birational to an abelian surface, then such $D_{+}$and $D_{-}$are unique up to $\mathbb{R}$-linearly equivalence classes.

(2) We set $D:=D_{+}+D_{-}$. Then $D$ is an $\mathbb{R}$-divisor on $X$ with $[D]=\nu$ that satisfies

$$
f^{*}(D)+f^{-1 *}(D) \sim_{\mathbb{R}}\left(\lambda+\lambda^{-1}\right) D .
$$

Proof. Since the assertion (2) follows from (1), it suffices to show (1).

Case 1: Suppose $X$ is not birational to an abelian surface. Then $X$ is either a rational surface, or a surface birational to a $K 3$ surface or an Enriques surface. In either case, we have $H^{1}\left(X, \mathcal{O}_{X}\right)=0$. Then the exponential sequence yields

$$
0 \longrightarrow \mathrm{Cl}(X) \stackrel{c_{1}}{\longrightarrow} H^{2}(X, \mathbb{Z}) .
$$

Hence $\mathrm{Cl}(X)_{\mathbb{R}} \cong N^{1}(X)_{\mathbb{R}}$ via $c_{1}$. Thus the existence and uniqueness of $D, D_{+}, D_{-}$follows.

Case 2: Suppose $X$ is birational to an abelian surface. We will show the existence of $D_{+}$. The existence of $D_{-}$is shown similarly. From the arguments in the proof of Lemma 2.2, we may assume that $X$ is an abelian surface.

Since $\lambda$ is an eigenvalue of $f^{*}: N^{1}(X) \rightarrow N^{1}(X), \lambda$ is a unit, i.e., both $\lambda$ and $\lambda^{-1}$ are algebraic integers. We put $k=[\mathbb{Q}(\lambda): \mathbb{Q}]$. Let

$$
S(t):=t^{k}+c_{1} t^{k-1}+\cdots+c_{k-1} t+c_{k}
$$

be the irreducible monic polynomial with $S(\lambda)=0$. By Theorem 2.1(2), the roots of $S(t)$ are given by

where $\left|\gamma_{i}\right|=1$

$$
\left\{\lambda, \lambda^{-1}, \gamma_{1}, \overline{\gamma_{1}}, \ldots, \gamma_{\frac{k}{2}-1}, \overline{\gamma_{\frac{k}{2}-1}}\right\}
$$

Since $\lambda$ is an eigenvalue of $f^{*}$, there is a nef class $\nu_{+}^{\circ} \in N^{1}(X) \otimes_{\mathbb{Z}} \mathbb{Z}[\lambda]$ with $f^{*}\left(\nu_{+}^{\circ}\right)=\lambda \nu_{+}^{\circ}$. Since $\nu_{+}=c \nu_{+}^{\circ}$ for some $c>0$, the existence of an $\mathbb{R}$-divisor $D_{+}^{\circ}$ for $\nu_{+}^{\circ}$ (i.e., $\left[D_{+}^{\circ}\right]=\nu_{+}^{\circ}$ and $\left.f^{*}\left(D_{+}^{\circ}\right) \sim_{\mathbb{R}} D_{+}^{\circ}\right)$ implies the existence of an $\mathbb{R}$-divisor $D_{+}$for $\nu_{+}$. Indeed, we just put $D_{+}:=c D_{+}^{\circ}$. Hence we may assume that $\nu_{+}=\nu_{+}^{\circ}$.

Since $\mathrm{Cl}(X) \rightarrow N^{1}(X)$ is surjective, we take $D_{+}^{\prime} \in \mathrm{Cl}(X) \otimes_{\mathbb{Z}} \mathbb{Z}[\lambda]$ with $\left[D_{+}^{\prime}\right]=\nu_{+}$. Let $\mathrm{Cl}^{0}(X)$ the kernel of $\mathrm{Cl}(X) \rightarrow N^{1}(X)$. Then $T:=f^{*}\left(D_{+}^{\prime}\right)-\lambda D_{+}^{\prime}$ belongs to $\mathrm{Cl}^{0}(X) \otimes_{\mathbb{Z}} \mathbb{Z}[\lambda]$.

Claim 3.8.1. The map $f^{*}-\lambda \mathrm{id}: \mathrm{Cl}^{0}(X) \otimes_{\mathbb{Z}} \mathbb{Z}[\lambda] \rightarrow \mathrm{Cl}^{0}(X) \otimes_{\mathbb{Z}} \mathbb{Z}[\lambda]$ is surjective.

We will show the claim at the end. For the moment, we admit the claim and proceed the proof. Admitting Claim 3.8.1, there is $T^{\prime} \in \mathrm{Cl}^{0}(X) \otimes_{\mathbb{Z}} \mathbb{Z}[\lambda]$ such that $f^{*}\left(T^{\prime}\right)-\lambda T^{\prime}=T$. We put $D_{+}:=D_{+}^{\prime}-T^{\prime}$. Then $\left[D_{+}\right]=\nu_{+}$and $f^{*}\left(D_{+}\right)-\lambda D_{+}=0 \in \operatorname{Cl}(X) \otimes_{\mathbb{Z}} \mathbb{Z}[\lambda]$. Hence we get the existence of $D_{+}$for $\nu_{+}$.

We are left to prove Claim 3.8.1. To see this, let us see the action of $f^{*}-\lambda$ id on $\mathrm{Cl}^{0}(X) \otimes_{\mathbb{Z}} \mathbb{Z}[\lambda]$ in more detail. We use the notation in Example 3.7.

Let $\bar{\Omega}:=\operatorname{Hom}_{\overline{\mathbb{C}}}\left(\mathbb{C}^{2}, \mathbb{C}\right)$ be the space of $\mathbb{C}$-antilinear forms $l: \mathbb{C}^{2} \rightarrow \mathbb{C}$. Let $\widehat{\Lambda}:=\{l \in$ $\bar{\Omega} \mid \operatorname{Im} l(\Lambda) \subseteq \mathbb{Z}\}$ be the dual lattice of $\Lambda$. The dual abelian surface of $X$ is defined by $\widehat{X}:=\bar{\Omega} / \widehat{\Lambda}$. By [4, Proposition 2.4.1], there is a canonical isomorphism $\widehat{X} \simeq \mathrm{Cl}^{0}(X)$. 
We define $\widehat{G}: \bar{\Omega} \rightarrow \bar{\Omega}$ by $l \mapsto l \circ G$. Then $\widehat{G}$ induces an automorphism $\widehat{g}: \widehat{X} \rightarrow \widehat{X}$. Then, by [4, Eqn. (1) in p. 35], one sees that $f^{*}=\widehat{g}$ under the canonical isomorphism $\mathrm{Cl}^{0}(X) \simeq \widehat{X}$. (We note that, for $L \in \mathrm{Cl}^{0}(X)$ and $v \in X(\mathbb{C})$, we have $t_{v}^{*}(L)=L$ by $[4$, Lemma 2.3.2, Proposition 2.4.1].)

We define the basis $\left\{l_{1}, l_{2}\right\}$ of $\bar{\Omega}=\operatorname{Hom}_{\overline{\mathbb{C}}}\left(\mathbb{C}^{2}, \mathbb{C}\right)$ by $l_{1}\left(z_{1}, z_{2}\right)=\overline{z_{1}}$ and $l_{2}\left(z_{1}, z_{2}\right)=\overline{z_{2}}$. Then $\widehat{G}$ is represented by the matrix ${ }^{t} \bar{P}=\left(\begin{array}{cc}\bar{p} & \bar{r} \\ \bar{q} & \bar{s}\end{array}\right)$.

To show the claim, it suffices to show

$$
\widehat{G} \otimes_{\mathbb{Z}} \mathbb{Z}[\lambda]-\lambda \mathrm{id}: \bar{\Omega} \otimes_{\mathbb{Z}} \mathbb{Z}[\lambda] \rightarrow \bar{\Omega} \otimes_{\mathbb{Z}} \mathbb{Z}[\lambda]
$$

is surjective. (Note that $\lambda$ in $\lambda$ id acts on $\bar{\Omega} \otimes_{\mathbb{Z}} \mathbb{Z}[\lambda]$ through $\mathbb{Z}[\lambda]$.) We take the $\mathbb{Z}$-basis $\left\{1, \lambda, \cdots, \lambda^{k-1}\right\}$ of $\mathbb{Z}[\lambda]$ over $\mathbb{Z}$. Then, with the basis $\left\{l_{1} \otimes 1, l_{2} \otimes 1, l_{1} \otimes \lambda, l_{2} \otimes \lambda, \ldots, l_{1} \otimes\right.$ $\left.\lambda^{k-1}, l_{2} \otimes \lambda^{k-1}\right\}$ of the $\mathbb{C}$-vector space $\bar{\Omega} \otimes_{\mathbb{Z}} \mathbb{Z}[\lambda], \widehat{G} \otimes_{\mathbb{Z}} \mathbb{Z}[\lambda]-\lambda$ id is represented by the $(2 k, 2 k)$ matrix

$$
M=\left(\begin{array}{ccccc}
{ }^{t} \bar{P} & 0 & \cdots & 0 & c_{k} I \\
-I & { }^{t} \bar{P} & \cdots & 0 & c_{k-1} I \\
\vdots & \ddots & \ddots & \vdots & \vdots \\
0 & 0 & \ddots & { }^{t} \bar{P} & c_{2} I \\
0 & 0 & \cdots & -I & { }^{t} \bar{P}+c_{1} I
\end{array}\right) \in M(2 k, 2 k ; \mathbb{C}) .
$$

We have $\operatorname{det} M=S(\bar{\alpha}) S(\bar{\beta})$, where $\bar{\alpha}, \bar{\beta}$ are the eigenvalues of ${ }^{t} \bar{P}$ with $|\bar{\alpha}|=\sqrt{\lambda}$ and $|\bar{\beta}|=\sqrt{\lambda^{-1}}$.

Since $\lambda, \lambda^{-1}$ are the only roots of $S(t)$ outside the unit circle, we get $S(\bar{\alpha}) \neq 0$ and $S(\bar{\beta}) \neq 0$. Hence $\operatorname{det} M \neq 0$. It follows that $\widehat{G} \otimes_{\mathbb{Z}} \mathbb{Z}[\lambda]-\lambda$ id is surjective.

Remark 3.9. If $\lambda$ is a quadratic irrational number, then, with a suitable $\nu, D$ can be taken as an integral divisor. (cf. Remark 2.6).

\section{Preliminaries on Height FunCtions}

In the latter half of this paper, we turn our attention to arithmetic properties. In this section, we briefly review some facts on height functions and fix notation. A general reference is $[12$, Part B] for example.

Let $K$ be a number field, and $O_{K}$ its ring of integers. For $x=\left(x_{0}: \cdots: x_{N}\right) \in \mathbb{P}^{N}(K)$, the logarithmic naive height of $x$ is defined by

$$
h_{n v}(x)=\frac{1}{[K: \mathbb{Q}]}\left[\sum_{P \in \operatorname{Spec}\left(O_{K}\right) \backslash\{0\}} \log \max _{i}\left\{\left\|x_{i}\right\|_{P}\right\}+\sum_{\sigma: K \hookrightarrow \mathbb{C}} \log \max _{i}\left\{\left|\sigma\left(x_{i}\right)\right|\right\}\right],
$$

where $\left\|x_{i}\right\|_{P}=\#\left(O_{K} / P\right)^{-\operatorname{ord}_{P}\left(x_{i}\right)}$. This definition naturally extends to all points $x \in \mathbb{P}^{N}(\overline{\mathbb{Q}})$, and gives rise to the logarithmic naive height function $h_{n v}: \mathbb{P}^{N}(\overline{\mathbb{Q}}) \rightarrow \mathbb{R}$.

We state Weil's height machine, which associates a height function to every $\mathbb{R}$-divisor, and summarize some properties of height functions. By a divisor we mean a Cartier divisor. For 
a proof, we refer the reader to [12, Theorem B.3.2]. See also [14, Theorems 1.1.1, 1.1.2]. In the following, $O(1)$ denotes a bounded function on $X(\bar{K})$.

Theorem 4.1 (cf. [12] Theorem B.3.2). Let $K$ be a number field. For any projective variety $X$ over $K$ and any $\mathbb{R}$-divisor $D$ on $X$, there is a way to attach a real-valued function, called a height function corresponding to $D$,

$$
h_{X, D}: X(\bar{K}) \rightarrow \mathbb{R}
$$

with the following properties.

(i) (Additivity) $h_{X, D_{1}+D_{2}}=h_{X, D_{1}}+h_{X, D_{2}}+O(1)$ for any $D_{1}, D_{2} \in \operatorname{Div}_{\mathbb{R}}(X)$.

(ii) (Normalization) If $H$ is a hyperplane in $\mathbb{P}^{N}$, then $h_{\mathbb{P}^{N}, H}=h_{n v}+O(1)$.

(iii) (Functoriality) If $f: X \rightarrow Y$ is a morphism of projective varieties and $D \in \operatorname{Div}_{\mathbb{R}}(Y$ ) with $f(X) \nsubseteq \operatorname{Supp}(D)$, then $h_{X, f^{*} D}=h_{Y, D} \circ f+O(1)$.

The height functions $h_{X, D}$ are uniquely determined, up to $O(1)$, by the above three properties. Moreover, they satisfy the following properties.

(iv) (Linear equivalence) If $D_{1}, D_{2} \in \operatorname{Div}_{\mathbb{R}}(X)$ are $\mathbb{R}$-linearly equivalent, then $h_{X, D_{1}}=$ $h_{X, D_{2}}+O(1)$.

(v) (Northcott finiteness property) Assume $D \in \operatorname{Div}_{\mathbb{R}}(X)$ is ample. Then for any positive integer $d$ and real number $M$, the set

$$
\left\{x \in X(\bar{K}) \mid[K(x): K] \leq d, h_{X, D}(x) \leq M\right\}
$$

is finite.

(vi) (Positivity) If $D \in \operatorname{Div}_{\mathbb{R}}(X)$ is effective, then there is a constant c such that $h_{X, D}(x) \geq$ $c$ for all $x \in(X \backslash \operatorname{Supp}(D))(\bar{K})$. If $D^{\prime} \in \operatorname{Div}_{\mathbb{R}}(X)$ is ample, then there is a constant $c^{\prime}$ such that $h_{X, D^{\prime}}(x) \geq c^{\prime}$ for all $x \in X(\bar{K})$.

(vii) If $D_{1}, D_{2} \in \operatorname{Div}_{\mathbb{R}}(X)$ are ample, then there are positive constants $a_{1}, a_{2}>0$ and constants $b_{1}, b_{2} \in \mathbb{R}$ such that

$$
a_{1} h_{X, D_{1}}(x)+b_{1} \leq h_{X, D_{2}}(x) \leq a_{2} h_{X, D_{1}}(x)+b_{2}
$$

for all $x \in X(\bar{K})$.

A function that coincides with $h_{X, D}$ up to $O(1)$ is also called a height function corresponding to $D$. In virtue of Theorem 4.1(iv), for a linear equivalence class $D \in \mathrm{Cl}(X) \otimes \mathbb{R}$, we define $h_{X, D}$ to be a height function corresponding an $\mathbb{R}$-divisor which represents $D$. We will often write $h_{D}$ in place of $h_{X, D}$ when $X$ is obvious from the context.

\section{Canonical height functions}

Let $X$ be a smooth projective surface over a number field $K \subset \mathbb{C}$, and $f: X \rightarrow X$ an automorphism over $K$. Let $f_{\mathbb{C}}: X_{\mathbb{C}} \rightarrow X_{\mathbb{C}}$ be the automorphism induced from $f$, where $f_{\mathbb{C}}:=f \times_{\operatorname{Spec}(K)} \operatorname{Spec}(\mathbb{C})$ and $X_{\mathbb{C}}:=X \times_{\operatorname{Spec}(K)} \operatorname{Spec}(\mathbb{C})$. We define the topological entropy of $f$ by that of $f_{\mathbb{C}}$.

Lemma 5.1. The $\mathbb{R}$-divisor classes $\nu, \nu_{+}, \nu_{-} \in N^{1}\left(X_{\mathbb{C}}\right)_{\mathbb{R}}$ in Proposition 2.5, the maximal $f$-invariant curve $E$ on $X_{\mathbb{C}}$ in Eqn. (3.2), and the $\mathbb{R}$-divisors $D, D_{+}, D_{-}$on $X_{\mathbb{C}}$ in Lemma 3.8 are all defined over $\bar{K}$. 
Proof. We set $X_{\bar{K}}:=X \times_{\operatorname{Spec}(K)} \operatorname{Spec}(\bar{K})$. We claim that $N^{1}\left(X_{\bar{K}}\right)=N^{1}\left(X_{\mathbb{C}}\right)$ naturally. Noting that a divisor refers to a Cartier divisor, $\mathrm{Cl}(S)$ is canonically isomorphic to $\operatorname{Pic}(S)$ for a projective scheme $S$ over a field. In this proof, we use Pic instead of $\mathrm{Cl}$.

Let $\mathrm{Pic}_{X_{\bar{K}} / \bar{K}}$ be the Picard scheme, and $\mathrm{Pic}_{X_{\bar{K}} / \bar{K}}^{0}$ the connected component of $\mathrm{Pic}_{X_{\bar{K}} / \bar{K}}$ that contains the trivial line bundle. In our case, $\mathrm{Pic}_{X_{\bar{K}} / \bar{K}}^{0}$ is also the irreducible component of $\mathrm{Pic}_{X_{\bar{K}} / \bar{K}}$ that contains the trivial line bundle.

By $\left[5, \S 8.1\right.$ Proposition 4], we have $\operatorname{Pic}_{X_{\bar{K}} / \bar{K}}(\bar{K})=\operatorname{Pic}\left(X_{\bar{K}}\right)$ and $\operatorname{Pic}_{X_{\bar{K}} / \bar{K}}(\mathbb{C})=\operatorname{Pic}\left(X_{\mathbb{C}}\right)$. Since, for an integral domain $A$ over $\bar{K}, A \otimes_{\bar{K}} \mathbb{C}$ is an integral domain, we have $\operatorname{Pic}_{X_{\bar{K}} / \bar{K}}^{0}(\bar{K})=$ $\operatorname{Pic}^{0}\left(X_{\bar{K}}\right)$ and $\operatorname{Pic}_{X_{\bar{K}} / \bar{K}}^{0}(\mathbb{C})=\operatorname{Pic}^{0}\left(X_{\mathbb{C}}\right)$. Hence $\operatorname{Pic}\left(X_{\bar{K}}\right) / \operatorname{Pic}^{0}\left(X_{\bar{K}}\right)=\operatorname{Pic}\left(X_{\mathbb{C}}\right) / \operatorname{Pic}^{0}\left(X_{\mathbb{C}}\right)(=$ $\left.\operatorname{Pic}_{X_{\bar{K}} / \bar{K}} / \operatorname{Pic}_{X_{\bar{K}} / \bar{K}}^{0}\right)$.

By Matsusaka's theorem [16], we have $N^{1}\left(X_{\bar{K}}\right)=\left(\operatorname{Pic}\left(X_{\bar{K}}\right) / \operatorname{Pic}^{0}\left(X_{\bar{K}}\right)\right) /($ torsion $)$ and $N^{1}\left(X_{\mathbb{C}}\right)=\left(\operatorname{Pic}\left(X_{\mathbb{C}}\right) / \operatorname{Pic}^{0}\left(X_{\mathbb{C}}\right)\right) /($ torsion $)$. Hence $N^{1}\left(X_{\bar{K}}\right)=N^{1}\left(X_{\mathbb{C}}\right)$. We then see that $\nu, \nu_{+}, \nu_{-}$in Proposition 2.5 are defined over $\bar{K}$.

Next, we show that $E$ is defined over $\bar{K}$. We fix an ample line bundle $\mathcal{O}(1)$ on $X_{\bar{K}}$ and let $P$ be the Hilbert polynomial of $E$. Let $\mathcal{H}:=\operatorname{Hilb}_{P}\left(X_{\bar{K}} / \bar{K}\right)$ be the Hilbert scheme. Let $\bar{e}: \operatorname{Spec}(\mathbb{C}) \rightarrow \mathcal{H}$ be the $\mathbb{C}$-valued point corresponding to $E$, and $e$ the scheme point, i.e., the image of $\bar{e}$ in $\mathcal{H}$. Let $\kappa$ be the residue field of $\mathcal{O}_{\mathcal{H}, e} . \operatorname{Via} \operatorname{Spec}(\mathbb{C}) \stackrel{\bar{e}}{\rightarrow} \mathcal{H} \rightarrow \operatorname{Spec}(\bar{K})$, we have $\bar{K} \subset \kappa \subset \mathbb{C}$. We claim that $\kappa=\bar{K}$. To see this, suppose $\kappa \neq \bar{K}$. Then there is an automorphism $\sigma: \mathbb{C} \rightarrow \mathbb{C}$ such that $\left.\sigma\right|_{K}=$ id and such that $\sigma(\kappa) \neq \kappa$. Let $\sigma^{*}: \operatorname{Spec}(\mathbb{C}) \rightarrow$ $\operatorname{Spec}(\mathbb{C})$ and $\sigma^{*}: X_{\mathbb{C}} \rightarrow X_{\mathbb{C}}$ be morphisms induced by $\sigma$. Since $E$ is the (unique) maximal $f$-invariant curve, we see that $\sigma^{*}(E)=E$. Hence $\bar{e} \circ \sigma^{*}=\bar{e}$, and $\sigma(\kappa)=\kappa$. This is a contradiction. Thus $\kappa=\bar{K}$, and $\bar{e}$ decomposes into $\operatorname{Spec}(\mathbb{C}) \rightarrow \operatorname{Spec}(\bar{K}) \rightarrow \mathcal{H}$. Hence $E$ is defined over $\bar{K}$.

Suppose $X$ is not birational to an abelian surface. Then $\operatorname{Cl}\left(X_{\bar{K}}\right) \simeq N^{1}\left(X_{\bar{K}}\right)$ implies that $D, D_{+}, D_{-}$in Lemma 3.8 are defined over $\bar{K}$.

Suppose now $X$ is birational to an abelian surface. We see that the morphism $f^{*}-\lambda$ id : $\operatorname{Pic}_{X_{\bar{K}} / \bar{K}}^{0} \otimes_{\mathbb{Z}} \mathbb{Z}[\lambda] \rightarrow \operatorname{Pic}_{X_{\bar{K}}}^{0} / \bar{K} \otimes_{\mathbb{Z}} \mathbb{Z}[\lambda]$ is surjective, because $f^{*}-\lambda \mathrm{id}: \operatorname{Pic}_{X_{\bar{K}} / \bar{K}}^{0}(\mathbb{C}) \otimes_{\mathbb{Z}} \mathbb{Z}[\lambda] \rightarrow$ $\operatorname{Pic}_{X_{\bar{K}} / \bar{K}}^{0}(\mathbb{C}) \otimes_{\mathbb{Z}} \mathbb{Z}[\lambda]$ is surjective by Claim 3.8.1. Hence $f^{*}-\lambda$ id : $\operatorname{Pic}^{0}\left(X_{\bar{K}}\right) \otimes_{\mathbb{Z}} \mathbb{Z}[\lambda] \rightarrow$ $\operatorname{Pic}^{0}\left(X_{\bar{K}}\right) \otimes_{\mathbb{Z}} \mathbb{Z}[\lambda]$ is surjective. A similar argument as in the proof of Lemma 3.8 shows that $D, D_{+}, D_{-}$are defined over $\bar{K}$.

In virtue of Lemma 5.1, we will often replace $K$ by its suitable finite extension so that $D$ and $E$ are defined over $K$.

Theorem 5.2. Let $X$ be a smooth projective surface over a number field $K \subset \mathbb{C}$, and $f: X \rightarrow X$ an automorphism of positive topological entropy $\log \lambda>0$. Let $D$ be a nef and big $\mathbb{R}$-divisor in Lemma 3.8, and $E$ the maximal $f$-invariant curve. Replacing $K$ by a suitable finite extension if necessary, we assume that $D$ and $E$ are defined over $K$. Then there exists a unique function

$$
\widehat{h}_{D}: X(\bar{K}) \rightarrow \mathbb{R}
$$

with the following two properties.

(i) $\widehat{h}_{D}$ is a height function corresponding to D. 
(ii) $\widehat{h}_{D}(f(x))+\widehat{h}_{D}\left(f^{-1}(x)\right)=\left(\lambda+\lambda^{-1}\right) \widehat{h}_{D}(x)$ for all $x \in X(\bar{K})$.

Moreover, $\widehat{h}_{D}$ satisfies the following properties.

(iii) $\widehat{h}_{D}(x) \geq 0$ for all $x \in X(\bar{K})$.

(iv) If $x \in E(\bar{K})$, then $\widehat{h}_{D}(x)=0$.

(v) Suppose $x \in(X \backslash E)(\bar{K})$. Then $\widehat{h}_{D}(x)=0$ if and only if $x$ is $f$-periodic.

(vi) $\widehat{h}_{D}$ satisfies the Northcott finiteness property on $(X \backslash E)(\bar{K})$ : For any positive integer $d$ and real number $M$, the set

$$
\left\{x \in(X \backslash E)(\bar{K}) \mid[K(x): K] \leq d, \widehat{h}_{D}(x) \leq M\right\}
$$

is finite.

We call $\widehat{h}_{D}$ a canonical height function for $f$ (and $D$ ).

Proof. (i) and (ii) This follows from a similar argument as in [21, Theorem 1.1]. Since $\lambda>1$ and $f^{*}(D)+f^{-1 *}(D) \sim_{\mathbb{R}}\left(\lambda+\lambda^{-1}\right) D$ by Lemma 3.8(2), Theorem 5.2(i)(ii) is also a special case of [14, Theorem 1.2.1], once we regard $\left(X ; f, f^{-1}\right)$ as a dynamical eigensystem for $D$ of degree $\left(\lambda+\lambda^{-1}\right)$.

For the sake of completeness, we briefly sketch a proof in line with [21]. Let $D_{+}, D_{-}$be as in Lemma 3.8, and $h_{D_{+}}, h_{D_{-}}$any height functions corresponding to $D_{+}, D_{-}$, respectively. Since

$$
f^{*}\left(D_{+}\right) \sim_{\mathbb{R}} \lambda D_{+} \quad \text { and } \quad f^{*}\left(D_{-}\right) \sim_{\mathbb{R}} \lambda^{-1} D_{-},
$$

Tate's telescoping argument asserts that the following limits exist:

$$
\widehat{h}_{D_{+}}(x):=\lim _{n \rightarrow+\infty} \frac{1}{\lambda^{n}} h_{D_{+}}\left(f^{n}(x)\right) \quad \text { and } \quad \widehat{h}_{D_{-}}(x):=\lim _{n \rightarrow+\infty} \frac{1}{\lambda^{n}} h_{D_{-}}\left(f^{-n}(x)\right) .
$$

Then $\widehat{h}_{D_{+}}$and $\widehat{h}_{D_{-}}$are height functions corresponding to $D_{+}$and $D_{-}$, which satisfy $\widehat{h}_{D_{+}} \circ f=$ $\lambda \widehat{h}_{D_{+}}$and $\widehat{h}_{D_{-}} \circ f=\lambda^{-1} \widehat{h}_{D_{-}}$, respectively. One defines

$$
\widehat{h}_{D}:=\widehat{h}_{D_{+}}+\widehat{h}_{D_{-}} .
$$

One can check that $\widehat{h}_{D}$ satisfies (i) and (ii). If $\widehat{h}_{D}$ and $\widehat{h}_{D}^{\prime}$ are two such functions, then $\widehat{h}_{D}-\widehat{h}_{D}^{\prime}$ is bounded and satisfies $\left(\lambda+\lambda^{-1}\right)\left\|\widehat{h}_{D}-\widehat{h}_{D}^{\prime}\right\|_{\text {sup }} \leq 2\left\|\widehat{h}_{D}-\widehat{h}_{D}^{\prime}\right\|_{\text {sup }}$, from which the uniqueness follows.

We remark that it follows from (5.1) that

$$
f^{n *}(D)+f^{-n *}(D) \sim_{\mathbb{R}}\left(\lambda^{n}+\lambda^{-n}\right) D
$$

for any $n \in \mathbb{Z}$. It follows from $\widehat{h}_{D_{ \pm}} \circ f=\lambda^{ \pm} \widehat{h}_{D_{ \pm}}$and (5.3) that

$$
\widehat{h}_{D}\left(f^{n}(x)\right)+\widehat{h}_{D}\left(f^{-n}(x)\right)=\left(\lambda^{n}+\lambda^{-n}\right) \widehat{h}_{D}(x)
$$

for any $n \in \mathbb{Z}$ and $x \in X(\bar{K})$. In the following, for clarity of the argument, we prove Theorem 5.2(iii)-(vi) in the order of (iv), (iii), (vi), (v).

(iv) To show (iv), we may replace $K$ by a suitable finite extension so that every component of $E$ is geometrically irreducible. Let $x \in E(\bar{K})$. Let $C$ be an irreducible component of $E$ such that $x \in C(\bar{K})$. Since $C$ is an $f$-periodic curve, there is a positive integer $n$ such that $f^{n}(C)=C$. We take $D^{\prime} \sim_{\mathbb{R}} D$ such that any component of $\operatorname{Supp}\left(D^{\prime}\right)$ is not equal to 
$\operatorname{Supp}(C)$, and we set $L:=\left.D^{\prime}\right|_{C}$. We take the normalization $\varphi: \widetilde{C} \rightarrow C$, set $\widetilde{L}:=\varphi^{*}(L)$, and write $\widetilde{f}^{n}: \widetilde{C} \rightarrow \widetilde{C}$ for the the automorphism induced by $f^{n}$. Then $\widetilde{L}$ is an $\mathbb{R}$-divisor on $C$ such that $\widetilde{f}^{n *}(\widetilde{L})+\widetilde{f}^{-n *}(\widetilde{L}) \sim_{\mathbb{R}}\left(\lambda^{n}+\lambda^{-n}\right) \widetilde{L}$. It follows from Lemma 5.3 below that $\widetilde{L} \sim_{\mathbb{R}} 0$.

We put $\widetilde{h}:=\left(\left.\widehat{h}_{D}\right|_{C}\right) \circ \varphi$. Since $\widetilde{h}$ is a height function corresponding to $\widetilde{L} \sim_{\mathbb{R}} 0, \widetilde{h}$ is a bounded function. Further, since $\widetilde{h}$ satisfies Eqn. (5.5) with $\widetilde{h}$ and $\widetilde{f}^{n}$ in place of $\widehat{h}_{D}$ and $f^{n}$, we conclude $\widetilde{h} \equiv 0$. Thus $\widehat{h}_{D}(x)=0$.

(iii) By (iv), we have only to show that $\widehat{h}_{D}(x) \geq 0$ for all $x \in(X \backslash E)(\bar{K})$. By Proposition 1.3 and Proposition 3.1, there is an effective divisor $Z$ with $\operatorname{Supp}(Z) \subseteq \operatorname{Supp}(E)$ such that $D-\varepsilon Z$ is ample for sufficiently small $\varepsilon>0$. Let $h_{Z}$ be a height function corresponding to $Z$. Then by Theorem $4.1(\mathrm{vi})$, there is constant $c_{1}$ such that

$$
\widehat{h}_{D}(x)-\varepsilon h_{Z}(x) \geq c_{1} \quad \text { for all } x \in X(\bar{K}) .
$$

Since $\operatorname{Supp}(Z) \subseteq \operatorname{Supp}(E)$, there is constant $c_{2}$ such that

$$
h_{Z}(x) \geq c_{2} \quad \text { for all } x \in(X \backslash E)(\bar{K}) .
$$

Note that $f$ induces the automorphism of $X \backslash E$. Then Eqn. (5.5) yields that, for any $x \in(X \backslash E)(\bar{K})$,

$$
\widehat{h}_{D}(x)=\frac{1}{\lambda^{n}+\lambda^{-n}}\left(\widehat{h}_{D}\left(f^{n}(x)\right)+\widehat{h}_{D}\left(f^{-n}(x)\right)\right) \geq \frac{1}{\lambda^{n}+\lambda^{-n}} 2\left(c_{1}+\varepsilon c_{2}\right) .
$$

Letting $n \rightarrow+\infty$, we get $\widehat{h}_{D}(x) \geq 0$ for $x \in(X \backslash E)(\bar{K})$.

(vi) If $x \in(X \backslash E)(\bar{K})$ satisfies $\widehat{h}_{D}(x) \leq M$, then $\widehat{h}_{D}(x)-\varepsilon h_{Z}(x) \leq M-\varepsilon c_{2}$ by Eqn. (5.7). Hence

$$
\begin{aligned}
\{x \in(X \backslash E)(\bar{K}) \mid[K(x): K] & \left.\leq d, \widehat{h}_{D}(x) \leq M\right\} \\
& \subseteq\left\{x \in X(\bar{K}) \mid[K(x): K] \leq d, \widehat{h}_{D}(x)-\varepsilon h_{Z}(x) \leq M-\varepsilon c_{2}\right\} .
\end{aligned}
$$

Since $\widehat{h}_{D}-\varepsilon h_{Z}$ is a height function corresponding to an ample $\mathbb{R}$-divisor, the latter set is a finite set by Theorem $4.1(\mathrm{v})$.

(v) Assume $x$ is $f$-periodic. Then $f^{n}(x)=x$ for some positive integer $n$. By Eqn. (5.5), we get $\widehat{h}_{D}(x)=0$. On the other hand, suppose $x \in(X \backslash E)(\bar{K})$ satisfies $\widehat{h}_{D}(x)=0$. Then by (ii) and (iii), we get $\widehat{h}_{D}\left(f^{n}(x)\right)=0$ for all $n \in \mathbb{Z}$. By (vi), we find that $\left\{f^{n}(x) \mid n \in \mathbb{Z}\right\}$ is a finite set. It follows that $x$ is an $f$-periodic point.

Lemma 5.3. Let $C$ be a smooth projective curve, $f$ an automorphism of $C$, and $\lambda>1$ a positive number. Suppose that an $\mathbb{R}$-divisor $L$ on $C$ satisfies

$$
f^{*}(L)+f^{-1 *}(L) \sim_{\mathbb{R}}\left(\lambda+\lambda^{-1}\right) L
$$

Then $L \sim_{\mathbb{R}} 0$.

Proof. By induction on $n$, we have $f^{n *}(L)+f^{-n *}(L) \sim_{\mathbb{R}}\left(\lambda^{n}+\lambda^{-n}\right) L$, so that we may freely replace $\lambda$ by $\lambda^{n}$ and $f$ by $f^{n}$ to prove the lemma.

Suppose that $g(C)=0$. Since $f^{*}(L) \sim_{\mathbb{R}} L$ and $f^{-1 *}(L) \sim_{\mathbb{R}} L$, Eqn. (5.8) implies that $\left(\lambda+\lambda^{-1}-2\right) L \sim_{\mathbb{R}} 0$. Hence $L \sim_{\mathbb{R}} 0$. 
Next, suppose that $g(C)=1$. We fix any point $O$ of $C$ and regard $C$ as an elliptic curve with the origin $O$. Since the set of the group isomorphisms of $C$ is a finite set, by replacing $f$ by a suitable $f^{n}$, we may assume that $f$ is a translation. By the theorem of the square, we then get $f^{*}(L)+f^{-1 *}(L) \sim_{\mathbb{R}} 2 L$. Thus $L \sim_{\mathbb{R}} 0$ from Eqn. (5.8).

Finally, suppose that $g(C) \geq 2$. Replacing $f$ by a suitable $f^{n}$, we may assume that $f=$ id. Then Eqn. (5.8) implies $L \sim_{\mathbb{R}} 0$.

Remark 5.4. In Example 3.5, let $\widehat{h}_{\text {Sil }}$ denote the canonical height function on $X$ over a number field, which is constructed in [21, Theorem 1.1]. As is checked in [14, Proposition 1.4.1], $\widehat{h}_{\text {Sil }}=(1+\sqrt{3}) \widehat{h}_{H_{1}+H_{2}}$.

We need in $\S 6.3$ the following proposition.

Proposition 5.5. Let the notation be as in Theorem 5.2. Let $\widehat{h}_{D_{+}}$and $\widehat{h}_{D_{-}}$as in Eqn. (5.2).

(1) $\widehat{h}_{D_{+}}(x) \geq 0$ and $\widehat{h}_{D_{-}}(x) \geq 0$ for all $x \in X(\bar{K})$.

(2) For $x \in(X \backslash E)(\bar{K})$, one has $\widehat{h}_{D_{+}}(x)=0 \Longleftrightarrow \widehat{h}_{D_{-}}(x)=0 \Longleftrightarrow \widehat{h}_{D}(x)=0$.

Proof. We follow [21].

(1) Since $\widehat{h}_{D}$ is non-negative by Theorem $5.2($ iii), we have, for any $x \in X(\bar{K})$,

$$
\widehat{h}_{D_{+}}(x)=\lambda^{-n} \widehat{h}_{D_{+}}\left(f^{n}(x)\right) \geq-\lambda^{-n} \widehat{h}_{D_{-}}\left(f^{n}(x)\right)=-\lambda^{-2 n} \widehat{h}_{D_{-}}(x) .
$$

Letting $n \rightarrow+\infty$, we get $\widehat{h}_{D_{+}}(x) \geq 0$. Similarly $\widehat{h}_{D_{-}}(x) \geq 0$.

(2) Let $L$ be an finite extension field of $K$ over which $x$ is defined. Suppose $\widehat{h}_{D_{+}}(x)=0$. Then we have

$$
\widehat{h}_{D}\left(f^{n}(x)\right)=\widehat{h}_{D_{+}}\left(f^{n}(x)\right)+\widehat{h}_{D_{-}}\left(f^{n}(x)\right)=\lambda^{-n} \widehat{h}_{D_{-}}(x) .
$$

Thus

$$
\left\{f^{n}(x) \mid n=1,2, \ldots,\right\} \subseteq\left\{z \in(X \backslash E)(L) \mid \widehat{h}_{D}(z) \leq \widehat{h}_{D_{-}}(x)\right\} .
$$

By Theorem 5.2(vi), $\left\{f^{n}(x) \mid n=1,2, \ldots,\right\}$ is a finite set. It follows that $x$ is $f$-periodic. Then Theorem 5.2(v) yields $\widehat{h}_{D}(x)=0$, so that $\widehat{h}_{D_{-}}(x)=0$. The other directions are shown similarly.

\section{Arithmetic properties of $f$}

6.1. Automorphisms of curves. In this subsection, we show some elementary arithmetic properties of automorphisms of curves, which will be needed in the sequel.

Let $f: \mathbb{P}^{1} \rightarrow \mathbb{P}^{1}$ be an automorphism over a number field $K$. Then there is a matrix $F=\left(\begin{array}{ll}p & q \\ r & s\end{array}\right) \in \mathrm{GL}_{2}(K)$ such that $f$ is given by $f(x: y)=(p x+q y: r x+s y)$ for $(x: y) \in \mathbb{P}^{1}(\bar{K})$. Let $P \in \mathrm{GL}_{2}(\bar{K})$ be a matrix such that $G:=P^{-1} F P \in \mathrm{GL}_{2}(\bar{K})$ is the Jordan canonical form of $F$. Then $G$ is one of the following types:

(i) $G=\left(\begin{array}{cc}\alpha & 0 \\ 0 & \beta\end{array}\right)$, where $\frac{\alpha}{\beta}$ is a root of unity;

(ii) $G=\left(\begin{array}{cc}\alpha & 0 \\ 0 & \beta\end{array}\right)$, where $\frac{\alpha}{\beta}$ is not a root of unity; 
(iii) $G=\left(\begin{array}{ll}\alpha & 1 \\ 0 & \alpha\end{array}\right)$ with $\alpha \in \bar{K} \backslash\{0\}$.

Let $p: \mathbb{P}^{1} \rightarrow \mathbb{P}^{1}$ be the automorphism induced from $P$, and $g: \mathbb{P}^{1} \rightarrow \mathbb{P}^{1}$ the automorphism induced from $G$.

As in $\S 4$, let $h_{n v}: \mathbb{P}^{1}(\bar{K}) \rightarrow \mathbb{R}$ denote the logarithmic naive height function.

First we consider $f$-periodic points.

Lemma 6.1. (1) If $G$ is of type (i), then $f$ is periodic, i.e., $f^{n}=\mathrm{id}$ for some $n \geq 1$.

(2) If $G$ is of type (ii), then the set of $f$-periodic points in $\mathbb{P}^{1}(\bar{K})$ consists of two points.

(3) If $G$ is of type (iii), then the set $f$-periodic points in $\mathbb{P}^{1}(\bar{K})$ consists of one point.

Proof. Since $f$ is conjugate to $g$, it suffices to show the lemma for $g$ in place of $f$. For $g$, the assertions are readily checked.

Next we consider non $f$-periodic points. We take a non $f$-periodic point $x \in \mathbb{P}^{1}(\bar{K})$. We write $O_{f}(x)=\left\{f^{n}(x) \mid n \in \mathbb{Z}\right\} \subset \mathbb{P}^{1}(\bar{K})$ for the orbit of $x$ under $f$. For $T>0$, we set

$$
N_{f, x}(T):=\#\left\{y \in O_{f}(x) \mid h_{n v}(y) \leq T\right\},
$$

which depends on $T, O_{f}(x)$ and $h_{n v}$.

For real-valued functions $k_{1}(T), k_{2}(T)$, we write

$$
k_{1}(T) \gg \ll k_{2}(T) \text { as } T \rightarrow+\infty
$$

if there are positive constants $a_{1}, a_{2}>0$ such that $a_{1} k_{1}(T) \leq k_{2}(T) \leq a_{2} k_{1}(T)$ for all sufficiently large $T$. As usual, we set $\log ^{+} x:=\log \max \{x, 1\}$ for a positive real number $x$.

Lemma 6.2. (1) If $G$ is of type (ii), then $N_{f, x}(T) \gg \ll T$ as $T \rightarrow+\infty$.

(2) If $G$ is of type (iii), then $N_{f, x}(T) \gg \ll \exp (T)$ as $T \rightarrow+\infty$.

Proof. To prove the lemma, we may replace $K$ by a finite extension field. So we may assume that $g: \mathbb{P}^{1} \rightarrow \mathbb{P}^{1}$ and $p: \mathbb{P}^{1} \rightarrow \mathbb{P}^{1}$ are defined over $K$. We take a finite extension field $L$ of $K$ over which $x$ is defined. We put $y=\left(y_{0}: y_{1}\right):=p^{-1}(x) \in \mathbb{P}^{1}(L)$.

By Theorem 4.1(iv), there is a constant $c$ such that $\left\|h_{n v}-h_{n v} \circ p\right\|_{\text {sup }} \leq c$ on $\mathbb{P}^{1}(\bar{K})$. Then

$$
\left|h_{n v}\left(f^{n}(x)\right)-h_{n v}\left(g^{n}(y)\right)\right|=\mid h_{n v}\left(p\left(g^{n}\left(p^{-1}(x)\right)\right)-h_{n v}\left(g^{n}(y)\right) \mid \leq c\right.
$$

for all $n \in \mathbb{Z}$. Hence we get $N_{g, y}(T-c) \leq N_{f, x}(T) \leq N_{g, y}(T+c)$. This estimate shows that it suffices to show the lemma for $g$ and $y$.

(1) We note $y_{0} y_{1} \neq 0$. Since $\frac{\alpha}{\beta}$ is not a root of unity, $h_{n v}((\alpha: \beta)) \neq 0$. Since $\left(\alpha^{n}: \beta^{n}\right)=$ $\left(\left(\frac{\alpha}{\beta}\right)^{n}: 1\right)$ and $g^{n}(y)=\left(\alpha^{n} y_{0}: \beta^{n} y_{1}\right)=\left(\left(\frac{\alpha}{\beta}\right)^{n} \frac{y_{0}}{y_{1}}: 1\right)$, we have

$$
\begin{aligned}
& h_{n v}\left(\left(\alpha^{n}: \beta^{n}\right)\right)=\frac{1}{[L: \mathbb{Q}]}\left[\sum_{P \in \operatorname{Spec}\left(O_{L}\right) \backslash\{0\}} \log ^{+}\left\|\left(\frac{\alpha}{\beta}\right)^{n}\right\|_{P}+\sum_{\sigma: L \hookrightarrow \mathbb{C}} \log ^{+}\left|\sigma\left(\left(\frac{\alpha}{\beta}\right)^{n}\right)\right|\right], \\
& h_{n v}\left(g^{n}(y)\right)=\frac{1}{[L: \mathbb{Q}]}\left[\sum_{P \in \operatorname{Spec}\left(O_{L}\right) \backslash\{0\}} \log ^{+}\left\|\left(\frac{\alpha}{\beta}\right)^{n} \frac{y_{0}}{y_{1}}\right\|_{P}+\sum_{\sigma: L \hookrightarrow \mathbb{C}} \log ^{+}\left|\sigma\left(\left(\frac{\alpha}{\beta}\right)^{n} \frac{y_{0}}{y_{1}}\right)\right|\right] .
\end{aligned}
$$


For any real numbers $a, b>0$, we have $\max \{a b, 1\} \leq \max \{a, 1\} \max \{b, 1\}$. Putting $a=\left(\frac{\alpha}{\beta}\right)^{n} \frac{y_{0}}{y_{1}}$ and $b=\left(\frac{y_{0}}{y_{1}}\right)^{-1}$ in Eqn. (6.1), and putting $a=\left(\frac{\alpha}{\beta}\right)^{n}$ and $b=\frac{y_{0}}{y_{1}}$ in Eqn. (6.2), we get

$$
h_{n v}\left(g^{n}(y)\right)-h_{n v}(y) \leq h_{n v}\left(\left(\alpha^{n}: \beta^{n}\right)\right)=|n| h_{n v}((\alpha: \beta)) \leq h_{n v}\left(g^{n}(y)\right)+h_{n v}(y) .
$$

Then $\lim _{T \rightarrow+\infty} \frac{N_{g, y}(T)}{T}=\frac{2}{h_{n v}((\alpha: \beta))}$. Hence $N_{g, y}(T) \gg \ll T$ as $T \rightarrow+\infty$.

(2) We note $y_{1} \neq 0$. Since $\frac{1}{\alpha} F \in \mathrm{GL}_{2}(K)$ is also a lift of $f: \mathbb{P}^{1} \rightarrow \mathbb{P}^{1}$, we may assume that $\alpha=1$. Then $g^{n}(y)=\left(y_{0}+n y_{1}: y_{1}\right)=\left(\frac{y_{0}}{y_{1}}+n: 1\right)$. We get

$$
h_{n v}\left(g^{n}(y)\right)=\frac{1}{[L: \mathbb{Q}]}\left[\sum_{P \in \operatorname{Spec}\left(O_{L}\right) \backslash\{0\}} \log ^{+}\left\|\frac{y_{0}}{y_{1}}+n\right\|_{P}+\sum_{\sigma: L \hookrightarrow \mathbb{C}} \log ^{+}\left|\sigma\left(\frac{y_{0}}{y_{1}}\right)+n\right|\right] \text {. }
$$

Since $\|n\|_{P} \leq 1$ for all $n$, we have $0 \leq \log ^{+}\left\|\frac{y_{0}}{y_{1}}+n\right\|_{P} \leq \log ^{+}\left\|\frac{y_{0}}{y_{1}}\right\|_{P}$. Also there is a constant $c^{\prime}$ (depending on $y$ ) such that $\left|\log ^{+}\right| \sigma\left(\frac{y_{0}}{y_{1}}\right)+n|-\log | n|| \leq c^{\prime}$ for all nonzero $n \in \mathbb{Z}$ and $\sigma$. Then there is a constant $c^{\prime \prime}$ (depending on $\left.y\right)$ such that $\left|h_{n v}\left(g^{n}(y)\right)-\log \right| n|| \leq c^{\prime \prime}$ for all nonzero $n \in \mathbb{Z}$. Hence $N_{g, y}(T) \gg \ll \exp (T)$ as $T \rightarrow+\infty$.

For automorphisms of curves in general, we have the following.

Lemma 6.3. Let $C$ be an geometrically irreducible curve over a number field $K$, and $f$ : $C \rightarrow C$ an automorphism over $K$. Let $H$ be an ample divisor on $C$, and we fix a height function $h_{H}$ corresponding to $H$. Then we have the following.

(1) Either (i) $f$ is periodic, or (ii) there are only finitely many f-periodic points on $C(\bar{K})$.

(2) In the case (ii), let $x \in C(\bar{K})$ be a non $f$-periodic point. Then

$$
\begin{aligned}
& \#\left\{y \in O_{f}(x) \mid h_{H}(y) \leq T\right\} \gg \ll T \quad \text { or } \quad \#\left\{y \in O_{f}(x) \mid h_{H}(y) \leq T\right\} \gg \ll \exp (T) \\
& \quad \text { as } T \rightarrow+\infty .
\end{aligned}
$$

Proof. Let $\varphi: \widetilde{C} \rightarrow C$ be the normalization, and $\underset{\widetilde{f}}{\widetilde{C}}: \widetilde{C} \rightarrow \widetilde{C}$ the automorphism induced by $f$. The assertion for $(C, f)$ follows from that for $(\widetilde{C}, \widetilde{f})$. Hence we may assume that $C$ is smooth.

Case 1: $g(C)=0$. This case is already treated in Lemma 6.1 and Lemma 6.2.

Case 2: $g(C)=1$. We fix any point $O$ of $C$ and regard $C$ as an elliptic curve with the origin $O$. As in the proof of Lemma 5.3, for some $n \in \mathbb{Z}_{>0}, f^{n}$ is a translation by a point $a \in C(\bar{K})$. If $a$ is a torsion point, then $f^{n m}=\mathrm{id}$ for some $m \in \mathbb{Z}_{>0}$, so that we have (i). If $a$ is not a torsion point, then $f^{n m}(x)=x+m a \neq x$ for any $x \in C(\bar{K})$ and for any $m \in \mathbb{Z}_{>0}$. This shows that there are no $f^{n}$-periodic points. Hence there are no $f$-periodic points, so that we have (ii).

Regarding (2), we will show that

$$
\#\left\{y \in O_{f}(x) \mid h_{H}(y) \leq T\right\} \gg \ll T \quad(T \rightarrow+\infty)
$$


for any non $f$-periodic point $x$. First we claim that we may replace $f$ by $f^{n}$. Indeed, suppose Eqn. (6.3) holds for $f^{n}$ and for any non $f^{n}$-periodic point. We will then have

$$
\begin{aligned}
\#\{y & \left.\in O_{f}(x) \mid h_{H}(y) \leq T\right\} \\
& =\sum_{i=0}^{n-1} \#\left\{y \in O_{f^{n}}\left(f^{i}(x)\right) \mid h_{H}(y) \leq T\right\} \gg \ll T \quad(T \rightarrow+\infty) .
\end{aligned}
$$

Hence, replacing $f$ by $f^{n}$, it suffices to show (6.3) when $f$ is a translation by $a$. Here $a$ is a non-torsion point, since we consider the case (ii). By Theorem 4.1(vii), we may assume that $h_{H}$ is the Néron-Tate height function $h_{N T}$ corresponding to an ample symmetric divisor. We set $2 B(x, y):=h_{N T}(x+y)^{2}-h_{N T}(x)^{2}-h_{N T}(y)^{2}$. Since $B$ is a bilinear form, we get

$$
h_{N T}\left(f^{n}(x)\right)=h_{N T}(x+n a)=\sqrt{h_{N T}(x)+2 n B(x, a)+n^{2} h_{N T}(a)} .
$$

Since $a$ is a non-torsion point, we have $h_{N T}(a) \neq 0$. Hence $\#\left\{y \in O_{f}(x) \mid h_{N T}(y) \leq T\right\} \gg \ll$ $T$ as $T \rightarrow+\infty$.

Case 3: $g(C) \geq 2$. Then $f^{n}=\mathrm{id}$ for some positive integer $n$. Thus we have (i).

6.2. $f$-periodic points. Let $f: X \rightarrow X$ be a smooth projective surface automorphism over a number field $K \subset \mathbb{C}$, which has positive topological entropy. Let $H$ be an ample line bundle on $X$, and $h_{H}$ a height function corresponding to $H$. A subset $S \subset X(\bar{K})$ is said to be of bounded height if there is a constant $c$ such that $h_{H}(s) \leq c$ for all $s \in S$.

Notice that, by Theorem 4.1(vii), the definition of a set of bounded height is independent of the choice of ample line bundles $H$. The Northcott finiteness property (Theorem 4.1(v)) asserts that if $S$ is a set of bounded height then $\{s \in S \mid[K(s): K] \leq d\}$ is finite for every positive integer $d$.

The set of all $f$-periodic points in $X(\bar{K})$ is infinite (cf. [7, Théorème in p. 906]). We would like to know if it is a set of bounded height. If there is an $f$-periodic curve $C$ such that $C$ is pointwisely fixed by $f^{n}$ for some positive integer $n$, then $\{f$-periodic points in $X(\bar{K})\}$ contains $C(\bar{K})$ and so is not a set of bounded height. On the other hand, the following theorem asserts that, if there are no such curves, then the set of all $f$-periodic points is of bounded height.

Theorem 6.4. Let $X$ be a smooth projective surface over a number field $K$, and $f: X \rightarrow X$ an automorphism of positive topological entropy. Let $E$ be the maximal $f$-invariant curve.

(1) The set $\{f$-periodic points in $(X \backslash E)(\bar{K})\}$ is of bounded height.

(2) Suppose that no $f$-periodic curve is pointwisely fixed by $f^{n}$ for each $n$. Then the set $\{f$-periodic points in $X(\bar{K})\}$ is of bounded height.

Proof. (1) As in the proof of Theorem 5.2(iii), let $h_{Z}$ be a height function corresponding to an effective divisor $Z$, where $Z$ satisfies $\operatorname{Supp}(Z) \subseteq \operatorname{Supp}(E)$ and $D-\varepsilon Z$ is ample for sufficiently small $\varepsilon>0$. Since $h_{Z}(x) \geq c_{2}$ for all $x \in(X \backslash E)(\bar{K})$ by Eqn. (5.7), we have

$$
\begin{aligned}
\{f \text {-periodic points in }(X \backslash E)(\bar{K})\} & =\left\{x \in(X \backslash E)(\bar{K}) \mid \widehat{h}_{D}(x)=0\right\} \\
& \subseteq\left\{x \in(X \backslash E)(\bar{K}) \mid\left(\widehat{h}_{D}-\varepsilon h_{Z}\right)(x) \leq-\varepsilon c_{2}\right\} .
\end{aligned}
$$


Since $\widehat{h}_{D}-\varepsilon h_{Z}$ is a height function corresponding to an ample $\mathbb{R}$-divisor, the latter set is a set of bounded height by Theorem 5.2 (vi).

(2) We use Lemma 6.3(1). The assumption of Theorem 6.4(2) says that the case (i) of Lemma 6.3(1) does not occur, so that $\{f$-periodic points in $E(\bar{K})\}$ is a finite set. Hence, trivially, $\{f$-periodic points in $E(\bar{K})\}$ is a set of bounded height. Now the assertion follows from (1).

6.3. Non $f$-periodic points. We keep the notation in $\S 6.2$. In particular, $h_{H}$ denotes a height function corresponding to an ample divisor $H$ on $X$.

Let $x$ be a non $f$-periodic point of $X(\bar{K})$. As in $\S 6.1$, let $O_{f}(x):=\left\{f^{n}(x) \mid n \in \mathbb{Z}\right\}$ be the orbit of $x$ under $f$. We set

$$
N_{f, x}(T):=\#\left\{z \in O_{f}(x) \mid h_{H}(z) \leq T\right\},
$$

which depends on $T, O_{f}(x)$ and $h_{H}$. We remark that we count $N_{f, x}(T)$ with respect to $h_{H}$ and not $\widehat{h}_{D}$.

Suppose further that $x$ does not lie on $E$. Then it follows from Theorem $5.2(\mathrm{v})$ and Proposition 5.5(2) that $\widehat{h}_{D_{+}}(z)>0$ and $\widehat{h}_{D_{-}}(z)>0$ for any $z \in O_{f}(x)$. Following [21], we set

$$
\widehat{h}_{D}\left(O_{f}(x)\right):=\frac{\log \left(\widehat{h}_{D_{+}}(z) \widehat{h}_{D_{-}}(z)\right)}{\log \lambda}
$$

for $z \in O_{f}(x)$. We see that $\widehat{h}_{D}\left(O_{f}(x)\right)$ is independent of the choice of $z \in O_{f}(x)$.

Theorem 6.5. Let the notation and assumption be as in Theorem 6.4. We take a non $f$-periodic point $x \in X(\bar{K})$. Then we have the following.

(1) (a) The general case: If $x$ is not on $E$, then $N_{f, x}(T) \gg \ll \log T$ as $T \rightarrow+\infty$.

(b) The special case: If $x$ is on $E$, then either $N_{f, x}(T) \gg \ll T$ or $N_{f, x}(T) \gg \ll$ $\exp (T)$ as $T \rightarrow+\infty$.

(2) In the case (a), we have a refined estimate in terms of $h_{\text {top }}(f)$ and $\widehat{h}_{D}$ :

$$
N_{f, x}(T)=\frac{2}{h_{t o p}(f)} \log T-\widehat{h}_{D}\left(O_{f}(x)\right)+O(1) \quad(T \rightarrow \infty),
$$

where the $O(1)$ constant is independent of $x$ (but depends on $h_{H}$ ).

Proof. (1)(a) This follows from (2) below.

(1)(b) Let $C_{0}$ be an irreducible $f$-periodic curve such that $x \in C_{0}(\bar{K})$. Let $k$ be the smallest positive integer such that $f^{k}\left(C_{0}\right)=C_{0}$. We set $C_{i}:=f^{i}\left(C_{1}\right)$ for $i=1, \ldots, k-1$. We also set $x_{0}:=x$ and $x_{i}:=f^{i}(x)$ for $i=1, \ldots, k-1$. Since $x$ is not $f$-periodic, as in (6.4) we get

$$
N_{f, x}(T)=\sum_{i=0}^{k-1} N_{f^{k}, x_{i}}(T) .
$$

By Theorem 4.1(vii), there are positive constants $a_{1}, a_{2}$ and constant $b_{1}, b_{2}$ such that $a_{1} h_{H}+$ $b_{1} \leq h_{n v} \leq a_{2} h_{H}+b_{2}$ on every $C_{i}$. Since $f^{k}\left(C_{i}\right)=C_{i}$ and $x_{i}$ is not $f$-periodic, Lemma 6.3 asserts that $N_{f^{k}, x_{i}}(T) \gg \ll T$ or $N_{f^{k}, x_{i}}(T) \gg \ll \exp (T)$ as $T \rightarrow+\infty$. Thus we get the assertion. 
(2) We can prove (2) as in [21, Theorem 1.3], together with comparison of $h_{H}$ and $\widehat{h}_{D}$. Indeed, we set

$$
\Sigma(T):=\#\left\{z \in O_{f}(x) \mid \widehat{h}_{D}(z) \leq T\right\} .
$$

Since $x$ is non $f$-periodic, we have

$$
\begin{aligned}
\Sigma(T) & =\#\left\{n \in \mathbb{Z} \mid \widehat{h}_{D}\left(f^{n}(x)\right) \leq T\right\} \\
& =\#\left\{n \in \mathbb{Z} \mid \lambda^{n} \widehat{h}_{D_{+}}(x)+\lambda^{-n} \widehat{h}_{D_{-}}(x) \leq T\right\} .
\end{aligned}
$$

It follows from $\left[21\right.$, Lemma in p. 366] that, if $T^{2} \geq 4 \widehat{h}_{D_{+}}(x) \widehat{h}_{D_{-}}(x)$, then

$$
-1 \leq \Sigma(T)-\frac{\log \frac{T^{2}}{4 \widehat{h}_{D_{+}}(x) \widehat{h}_{D_{-}}(x)}}{\log \lambda} \leq 1+\frac{\log 4}{\log \lambda} .
$$

Since $h_{\mathrm{top}}(f)=\log \lambda$ by Theorem 2.1(1), we get

$$
\Sigma(T)=\frac{2}{h_{\text {top }}(f)} \log T-\widehat{h}_{D}\left(O_{f}(x)\right)+O(1) \quad(T \rightarrow+\infty) .
$$

Since $h_{H}$ is a height function corresponding to an ample $\mathbb{R}$-divisor, there is a positive constant $a_{1}>0$ and a constant $b_{1}$ such that

$$
h_{H}(x) \geq a_{1} \widehat{h}_{D}(x)+b_{1} \quad \text { for all } x \in X(\bar{K}) .
$$

On the other hand, by Proposition 1.3(2) and Eqn. (5.7), there is a positive constant $a_{2}>0$ and a constant $b_{2}$ such that

$$
h_{H}(x) \leq a_{2} \widehat{h}_{D}(x)+b_{2} \quad \text { for all } x \in(X \backslash E)(\bar{K}) .
$$

Since $x$ is not on $E$, we have $O_{f}(x) \subset(X \backslash E)(\bar{K})$. Now the assertion follows from Eqns. (6.5), (6.6) and (6.7).

For $x \in X(\bar{K})$, let $O_{f}^{+}(x):=\left\{f^{n}(x) \mid n \in \mathbb{Z}_{\geq 0}\right\}$ denote the set of forward-orbit of $x$ under $f$. If $x$ is not $f$-periodic, we set $N_{f, x}^{+}(T):=\#\left\{z \in O_{f}^{+}(x) \mid h_{H}(z) \leq T\right\}$. Then we have the following corollary.

Corollary 6.6. If $x \in(X \backslash E)(\bar{K})$ is not f-periodic, then

$$
\lim _{n \rightarrow+\infty} \frac{N_{f, x}^{+}(T)}{\log T}=\frac{1}{h_{\text {top }}(f)} .
$$

Proof. For $T \gg 1$, we set

$$
S(T):=\left\{n \in \mathbb{Z}_{\geq 0} \mid \lambda^{n} \widehat{h}_{D_{+}}(z)+\lambda^{-n} \widehat{h}_{D_{-}}(z) \leq T\right\} .
$$

If $n \in S(T)$, then $\lambda^{n} \widehat{h}_{D_{+}}(z) \leq T$. Thus $\# S(T) \leq 2+\frac{\log T-\log \widehat{h}_{D_{+}}(z)}{\log \lambda}$. On the other hand, if $n \in$ $\mathbb{Z}_{\geq 0}$ satisfies $\lambda^{n} \widehat{h}_{D_{+}}(z)+\widehat{h}_{D_{-}}(z) \leq T$, then $n \in S(T)$. Thus $\# S(T) \geq \frac{\log \left(T-\widehat{h}_{D_{-}}(z)\right)-\log \widehat{h}_{D_{+}}(z)}{\log \lambda}$. By Eqns. (6.6) and (6.7), we get the assertion. 
SHU KAWAGUCHI

\section{REFERENCES}

[1] A. Baragar, Rational points on K3 surfaces in $\mathbb{P}^{1} \times \mathbb{P}^{1} \times \mathbb{P}^{1}$, Math. Ann. 305 (1996), 541-558.

[2] W. Barth, K. Hulek, C. Peters, A. Van de Ven, Compact complex surfaces, Second edition, Springer, 2004.

[3] A. Beauville, Complex algebraic surfaces, Second edition, Cambridge Univ. Press, 1996.

[4] C. Birkenhake and H. Lange, Complex abelian varieties, Second edition, Springer, 2004.

[5] S. Bosch, W. Lütkebohmert and M. Raynaud, Néron models, Springer, 1990.

[6] G. S. Call and J. H. Silverman, Canonical heights on varieties with morphisms, Compositio Math. 89 (1993), 163-205.

[7] S. Cantat, Dynamique des automorphismes des surfaces projectives complexes, C. R. Acad. Sci. Paris Sér. I Math. 328 (1999), 901-906.

[8] S. Cantat, Dynamique des automorphismes des surfaces K3, Acta Math. 187 (2001), 1-57.

[9] J. Diller and C. Favre, Dynamics of bimeromorphic maps of surfaces, Amer. J. Math. 123 (2001), $1135-1169$.

[10] T.-C. Dinh and N. Sibony, Groupes commutatifs d'automorphismes d'une variété kählérienne compacte, Duke Math. J. 123 (2004), 311-328.

[11] R. Hartshorne, Algebraic geometry, GTM 52, Springer, 1977.

[12] M. Hindry and J. H. Silverman, Diophantine geometry, Springer, 2000.

[13] S. Kawaguchi, Canonical height functions for affine plane automorphisms, Math. Ann. 335 (2006), $285-310$.

[14] S. Kawaguchi, Canonical heights, invariant currents, and dynamical eigensystems of morphisms for line bundles, J. Reine Angew. Math. 597 (2006), 135-173.

[15] R. Lazarsfeld, Positivity in algebraic geometry. I, Springer, 2004.

[16] T. Matsusaka, The criteria for algebraic equivalence and the torsion group, Amer. J. Math. 79 (1957), $53-66$.

[17] B. Mazur, The topology of rational points, Experiment. Math. 1 (1992), 35-45.

[18] C. T. McMullen, Dynamics on K3 surfaces: Salem numbers and Siegel disks, J. Reine Angew. Math. 545 (2002), 201-233.

[19] K. Oguiso, Automorphisms of hyperkähler manifolds in the view of topological entropy, Algebraic geometry, 173-185, Contemp. Math. 422, Amer. Math. Soc., Providence, RI, 2007.

[20] I. I. Pjateckiı̌-Šapiro and I. R. Šafarevič, Torelli's theorem for algebraic surfaces of type K3, Izv. Akad. Nauk SSSR Ser. Mat. 35 (1971), 530-572.

[21] J. Silverman, Rational points on K3 surfaces: a new canonical height, Invent. Math. 105 (1991), 347373.

[22] L. Wang, Rational points and canonical heights on K3-surfaces in $\mathbb{P}^{1} \times \mathbb{P}^{1} \times \mathbb{P}^{1}$, in Recent developments in the inverse Galois problem (Seattle, WA, 1993), Contemp. Math. 186 (1995), 273-289.

Department of Mathematics, Faculty of Science, Kyoto University, Kyoto, 606-8502, JAPAN

E-mail address: kawaguch@math.kyoto-u.ac.jp 